\title{
Theoretical and numerical local null controllability of a Ladyzhenskaya-Smagorinsky model of turbulence
}

\author{
E. Fernández-Cara, J. Limaco, S. B. de Menezes
}

\begin{abstract}
This paper deals with the control of a differential turbulence model of the Ladyzhenskaya-Smagorinsky kind. In the equations we find local and nonlocal nonlinearities: the usual transport terms and a turbulent viscosity that depends on the global in space energy dissipated by the mean flow. We prove that the system is locally null-controllable, with distributed controls locally supported in space. The proof relies on rather well known arguments. However, some specific difficulties are found here because of the occurrence of nonlocal nonlinear terms. We also present an iterative algorithm of the quasi-Newton kind that provides a sequence of states and controls that converge towards a solution to the control problem. Finally, we give the details of a numerical approximation and we illustrate the behavior of the algorithm with a numerical experiment.
\end{abstract}

\section{Introduction and main results}

Let $\Omega \subset \mathbb{R}^{N}$ ( $N=2$ or 3$)$ be a non-empty open bounded connected set, with $C^{2}$ boundary $\partial \Omega$ and let us set $Q=\Omega \times(0, T)$, where $T>0$. The lateral boundary of $Q$ is $\Sigma=\partial \Omega \times(0, T)$ and we denote by $n(x)$ the outward unit normal to $\Omega$ at the point $x \in \partial \Omega$.

In the sequel, we denote by $(\cdot, \cdot)$ and $\|\cdot\|$ respectively the $L^{2}$ scalar product and norm in $\Omega$. The symbol $C$ is used to design a generic positive constant.

Let $\omega \subset \Omega$ be a non-empty open set. We deal with the null controllability of the nonlinear system

$$
\begin{cases}y_{t}-\nabla \cdot\left(\left(\nu_{0}+\nu_{1}\left(\|D y\|^{2}\right)\right) D y\right)+(y \cdot \nabla) y+\nabla p=v 1_{\omega} & \text { in } Q, \\ \nabla \cdot y=0 & \text { in } Q, \\ y=0 & \text { on } \Sigma, \\ y(x, 0)=y_{0}(x) & \text { in } \Omega .\end{cases}
$$

Here, $y=y(x, t)$ and $p=p(x, t)$ represent the "averaged" velocity field and pressure of a turbulent fluid whose particles are in $\Omega$ during the time interval $(0, T) ; y_{0}$ is the averaged velocity at time $t=0 ; 1_{\omega}$ is the characteristic function of $\omega ; \nu_{0}$ is a positive constant (the kinematic viscosity of the fluid); $\nu_{1} \in C_{b}^{1}(\mathbb{R})$ with $\nu_{1} \geq 0$ (that is, $\nu_{1}$ is continuously differentiable, $0 \leq \nu_{1} \leq C$ 
and $\left|\nu_{1}^{\prime}\right| \leq C ; \nu_{1}$ is the so called turbulent viscosity) and $D y$ stands for the symmetrized gradient of $y: D y=\nabla y+\nabla^{T} y$.

On the other hand, $\omega \times(0, T)$ is the control domain and $v$ must be viewed as a control (an averaged force) acting on the system.

The nonlocal term in (1) has an important physical motivation. Indeed, it is the result of assuming that the following Boussinesq hypothesis holds:

$$
R=\nu_{1}\left(\|D y\|^{2}\right) D y
$$

where $R$ is the Reynolds tensor; see for instance 5, 23, 28,

The following vector spaces, usual in the context of incompressible fluids, will be used along the paper:

$$
H=\left\{w \in L^{2}(\Omega)^{N}: \nabla \cdot w=0 \text { in } \Omega, w \cdot n=0 \text { on } \partial \Omega\right\}
$$

and

$$
V=\left\{w \in H_{0}^{1}(\Omega)^{N}: \nabla \cdot w=0 \text { in } \Omega\right\} .
$$

We will denote by $A: D(A) \mapsto H$ the Stokes operator. By definition, one has

$$
D(A)=H^{2}(\Omega)^{N} \cap V, \quad A w=P(-\Delta w) \quad \forall w \in D(A),
$$

where $P: L^{2}(\Omega)^{N} \mapsto H$ is the usual orthogonal projector.

When $N=2$, for any $y_{0} \in V$ and any $v \in L^{2}(\omega \times(0, T))$, (1) possesses exactly one strong solution $(y, p)$, with

$$
y \in L^{2}(0, T ; D(A)) \cap C^{0}([0, T] ; V), \quad y_{t} \in L^{2}(0, T ; H) .
$$

When $N=3$, this is true if $y_{0}$ and $v$ are sufficiently small in their respective spaces.

These assertions can be deduced arguing (for instance) as in [11; see the main ideas of the proofs in Appendix A.

Definition 1.1. It will be said that (1) is (globally) null-controllable at time $T$ if, for any $y_{0} \in V$, there exist controls $v \in L^{2}(\omega \times(0, T))^{N}$ and associated states $y$ satisfying (2) and

$$
y(x, T)=0 \text { in } \Omega .
$$

On the other hand, it will be said that (1) is locally null-controllable at time $T$ if there exists $\epsilon>0$ such that, for any $y_{0} \in V$ with

$$
\left\|y_{0}\right\|_{H_{0}^{1}} \leq \epsilon
$$

there exist controls $v \in L^{2}(\omega \times(0, T))^{N}$ and associated states $y$ satisfying (2) and $(3)$.

Recently, important progress has been made in the controllability analysis of linear and nonlinear parabolic equations and systems; we refer to the works [8, 13, 20, 24, 30, 31, 32 and the references therein; in particular, for the NavierStokes system, the first null controllability results have been established by Fursikov and Imanuvilov in 19 and Fursikov in [18. Consequently, it is natural to try to extend the known results to systems like (1).

In this paper, the first main result is the following: 
Theorem 1.1. The nonlinear system (1) is locally null-controllable at any $T>$ 0 .

We will employ some techniques relying on the so called Liusternik's Inverse Mapping Theorem in Hilbert spaces, see [1. The arguments are inspired by the work of Fursikov and Imanuvilov, see [19-20. See also [6, for some similar results concerning other control systems with local and nonlocal nonlinearities.

Thus, in a first step, we consider a linearized system at zero

$$
\begin{cases}y_{t}-\left(\nu_{0}+\nu_{1}(0)\right) \Delta y+\nabla p=v 1_{\omega}+f & \text { in } Q, \\ \nabla \cdot y=0 & \text { in } Q, \\ y=0 & \text { on } \Sigma, \\ y(x, 0)=y_{0}(x) & \text { in } \Omega .\end{cases}
$$

The adjoint of $(5)$ is given by

$$
\begin{cases}-\varphi_{t}-\left(\nu_{0}+\nu_{1}(0)\right) \Delta \varphi+\nabla \pi=F & \text { in } Q, \\ \nabla \cdot \varphi=0 & \text { in } Q, \\ \varphi=0 & \text { on } \Sigma, \\ \varphi(x, T)=\varphi_{T}(x) & \text { in } \Omega .\end{cases}
$$

Following well known ideas, the null controllability of (5) is obtained as a consequence of suitable Carleman estimates for the solutions to (6) with arbitrary final data $\varphi_{T}$.

In a second step, following the ideas introduced by Fursikov and Imanuvilov, we rewrite the null controllability property of (1) as an equation of the form

$$
\mathcal{A}(y, p, v)=\left(0, y_{0}\right)
$$

in a well chosen space $E_{N}$ of "admissible" state-controls; see (28) and (29). In fact, the definitions of $E_{N}$ and $\mathcal{A}: E_{N} \mapsto F_{N}$ are nontrivial. Below, we make a particular choice that allows $\mathcal{A}$ to be well defined and $C^{1}$ near zero (to this purpose, $E_{N}$ and $F_{N}$ must be large enough) and, also, allows $\mathcal{A}^{\prime}(0,0,0)$ to be onto (accordingly, $E_{N}$ and $F_{N}$ cannot be too large). Actually, the surjectivity of $\mathcal{A}^{\prime}(0,0,0)$ is, in a certain sense, equivalent to the (global) null controllability of (5).

Due to the nonlocal nonlinear term in (5), we find some difficulties in this process that are new with respect to a similar argument for the classical NavierStokes equations. Indeed, in order to ensure that, for any $(y, p, v) \in E_{N}$, $\mathcal{A}(y, p, v)$ makes sense and belongs to an appropriate space, we have to estimate the growth of $\left(\nu_{1}\left(\|D y\|^{2}\right)-\nu_{1}(0)\right) \Delta y(\cdot, t)$ as $t \rightarrow T$. This needs some previous work: see Propositions 2.2 and 2.3, used in the proofs of Lemmas 3.1 and 3.2 .

Then, we apply Liusternik's Theorem (see Theorem 3.1) and we deduce the (local) desired result.

Notice that the null controllability of (5) can also be proved using other relatively standard arguments. 
In particular, we can try to use a fixed point technique. Roughly speaking, the main task would be to get a sufficiently regular fixed point of the mapping $z \mapsto y$, where $y, p$, and $v$ satisfy

$$
\begin{cases}y_{t}-\mu(t) \Delta y+(z \cdot \nabla) y+\nabla p=v 1_{\omega} & \text { in } Q, \\ \nabla \cdot y=0 & \text { in } Q, \\ \text { etc. } & \end{cases}
$$

and (3) and $\mu=\nu_{0}+\nu_{1}\left(\|D z\|^{2}\right)$. In view of the particular structure of this system, we can perform a change of variable that leads to the following equivalent form:

$$
\begin{cases}y_{s}-\Delta y+m(s)(z \cdot \nabla) y+\nabla(m(s) p)=m(s) v 1_{\omega} & \text { in } \Omega \times(0, S), \\ \nabla \cdot y=0 & \text { in } \Omega \times(0, S), \\ \text { etc. } & \end{cases}
$$

for some $S>0$ and some $m \in C^{0}([0, S])$ that is bounded from above and from below by positive constants. Consequently, we can apply some already known results on the null controllability of Stokes and Navier-Stokes systems to achieve the proof.

However, we have preferred a strategy relying on Liusternik's Theorem for the following reasons:

- The fixed point argument needs the compactness of the mapping $z \mapsto$ $y$ in a space ensuring (at least) the continuity of $\mu$. Essentially, this means compactness in $C^{0}([0, T] ; V)$ and, accordingly, we have to be able to control (7) with controls in $L^{\infty}\left(0, T ; L^{2}(\omega)^{N}\right)$, which is a little more involved from the technical viewpoint.

- Our strategy can be generalized to cover other interesting PDEs with more general nonlocal nonlinearities. For example, it can be applied to the system

$$
\begin{cases}y_{t}-\nabla \cdot\left(M\left(\|\nabla y\|^{2}\right) D y\right)+(z \cdot \nabla) y+\nabla p=v 1_{\omega} & \text { in } Q, \\ \nabla \cdot y=0 & \text { in } Q, \\ \text { etc. } & \end{cases}
$$

where $M=M(r)$ is an appropriate (matrix-valued) viscosity tensor, which can be used to model non-isotropic turbulence, see for instance $\underline{3.27}$.

- Moreover, our approach leads to a formulation of the null controllability problem whose solution can be approximated by the iterates of a convergent algorithm, see below.

We are also interested in this paper by the computation of "good" null controls. To this end, we will introduce below an iterative algorithm of the quasi-Newton kind:

\section{ALG 1:}


1. Choose $\left(y^{0}, p^{0}, v^{0}\right) \in E_{N}$.

2. Then, for given $n \geq 0$ and $\left(y^{n}, p^{n}, v^{n}\right) \in E_{N}$, compute

$$
\left(y^{n+1}, p^{n+1}, v^{n+1}\right)=\left(y^{n}, p^{n}, v^{n}\right)-\mathcal{B}\left(\mathcal{A}\left(y^{n}, p^{n}, v^{n}\right)-\left(0, y_{0}\right)\right) .
$$

Here, $\mathcal{B}$ is an inverse to $\mathcal{A}^{\prime}(0,0,0)$, whose precise definition is furnished in Section 3 .

A good property of ALG $\mathbf{1}$ is that, after discretization, it leads at each step to a linear algebraic system where the coefficient matrix is always the same. Of course, this is very convenient from the numerical viewpoint, since it makes it possible to perform just one matrix factorization at the first iterate and then compute very quickly every $\left(y^{n+1}, p^{n+1}, v^{n+1}\right)$.

Our second main result is the following:

Theorem 1.2. Let $\epsilon$ be the quantity furnished by Theorem 1.1 that ensures null controllability for any initial state satisfying (4). Assume that $\left\|y_{0}\right\|_{H_{0}^{1}} \leq \epsilon$, $(y, p, v)$ satisfies (1) and (3) and $\|(y, p, v)\|_{E_{N}}$ is sufficiently small. There exists $\kappa>0$ such that, if $\left(y^{0}, p^{0}, v^{0}\right) \in E_{N}$ and

$$
\left\|\left(y^{0}, p^{0}, v^{0}\right)-(y, p, v)\right\|_{E_{N}} \leq \kappa,
$$

then the $\left(y^{n}, p^{n}, v^{n}\right)$ converge to $(y, p, v)$ and satisfy

$$
\left\|\left(y^{n+1}, p^{n+1}, v^{n+1}\right)-(y, p, v)\right\|_{E_{N}} \leq \theta\left\|\left(y^{n}, p^{n}, v^{n}\right)-(y, p, v)\right\|_{E_{N}}
$$

for some $\theta \in(0,1)$ for all $n \geq 0$.

This convergence result is illustrated in Section 5 with some numerical experiments.

This paper is organized as follows.

In Section 2, we prove some technical results and we establish the null controllabilty of (5).

In Section 3 , we give the proof of Theorem 1.1.

Section 4 is devoted to the formulation of ALG 1 and the proof of Theorem 1.2 ,

We exhibit some numerical results in Section 5. Finally, some additional comments and questions are presented in Section 6 .

\section{Preliminary results}

\subsection{Some Carleman estimates}

In the sequel, we will set $\nu:=\nu_{0}+\nu_{1}(0)$.

We will need some results from [20, [14, 25 and 26]. Also, it will be convenient to introduce a new non-empty open set $\omega_{0}$, with $\omega_{0} \Subset \omega$. The following technical result, due to Fursikov and Imanuvilov 20, is fundamental: 
Lemma 2.1. There exists a function $\eta^{0} \in C^{2}(\bar{\Omega})$ satisfying:

$$
\left\{\begin{array}{l}
\eta^{0}(x)>0 \quad \forall x \in \Omega, \quad \eta^{0}(x)=0 \quad \forall x \in \partial \Omega \quad \text { and } \\
\left|\nabla \eta^{0}(x)\right|>0 \quad \forall x \in \bar{\Omega} \backslash \omega_{0} .
\end{array}\right.
$$

Let $\tau=\tau(t)$ be a function satisfying

$$
\tau \in C^{\infty}([0, T]), \quad \tau>0 \quad \text { in }(0, T), \quad \tau(t)= \begin{cases}t & \text { if } t \leq T / 4, \\ T-t & \text { if } t \geq 3 T / 4\end{cases}
$$

and let us introduce the functions

$$
\left\{\begin{array}{l}
\alpha(x, t):=\frac{e^{\lambda\left(\left\|\eta^{0}\right\|_{\infty}+m_{0}+1\right)}-e^{\lambda\left(\eta^{0}(x)+m_{0}\right)}}{\tau(t)^{8}}, \quad \xi(x, t):=\frac{e^{\lambda\left(\eta^{0}(x)+m_{0}\right)}}{\tau(t)^{8}}, \\
\rho(x, t):=e^{s \alpha(x, t)}, \quad \bar{\rho}(t):=\exp \left(s \max _{x \in \bar{\Omega}} \alpha(x, t)\right), \quad \bar{\xi}(t):=\max _{x \in \bar{\Omega}} \xi(x, t),
\end{array}\right.
$$

where $\lambda, s>0$ and the constant $m_{0}>0$ is fixed, sufficiently large to have $\left|\alpha_{t}\right| \leq C \xi^{9 / 8}$ and $\left|\alpha_{t t}\right| \leq C \xi^{5 / 4}$ for all $\lambda>0$.

There exists $\lambda_{00}>0$ such that, for any $\lambda \geq \lambda_{00}$, one has

$$
\max _{\bar{\Omega}} \alpha_{0}(x)<2 \min _{\bar{\Omega}} \alpha_{0}(x) .
$$

In the sequel, for future purposes, we will always take $\lambda \geq \lambda_{00}$.

The following global Carleman estimates hold for the solutions to (6):

Lemma 2.2. There exists $\lambda_{0}>0$ such that, for each $\lambda \geq \lambda_{0}$, there exist $s_{0}$ and $C$ with the following property: for any $F \in L^{2}(Q)^{N}$, any $\varphi_{T} \in H$ and any $s \geq s_{0}$, the corresponding solution to (6) satisfies

$$
\begin{gathered}
\iint_{Q} \bar{\rho}^{-2}(s \bar{\xi})^{-1}\left(|\varphi t|^{2}+|\nabla \pi|^{2}\right) d x d t+\iint_{Q} \rho^{-2}(s \xi)|\nabla \times \varphi|^{2} d x d t \\
\quad+\iint_{Q} \rho^{-2}\left((s \xi)^{-1}|\Delta \varphi|^{2}+|\nabla \varphi|^{2}+(s \xi)^{2}|\varphi|^{2}\right) d x d t \\
\leq C\left(\iint_{Q} \rho^{-2}|F|^{2} d x d t+\iint_{\omega \times(0, T)} \rho^{-2}(s \xi)^{3}|\varphi|^{2} d x d t\right) .
\end{gathered}
$$

The proof relies on the results and ideas in 25] and 26]. For clarity, the main steps are sketched in Appendix B.

Let us introduce the function $\ell=\ell(t)$, with

$$
\ell(t):=\left\{\begin{array}{l}
\tau(T / 2) \text { for } 0 \leq t \leq \frac{T}{2}, \\
\tau(t) \text { for } \frac{T}{2} \leq t \leq T
\end{array}\right.
$$


and let us set

$$
\left\{\begin{array}{l}
\tilde{\alpha}(x, t):=\frac{e^{\lambda\left(\left\|\eta^{0}\right\|_{\infty}+m_{0}+1\right)}-e^{\lambda\left(\eta^{0}(x)+m_{0}\right)}}{\ell(t)^{8}}, \tilde{\xi}(x, t):=\frac{e^{\lambda\left(\eta^{0}(x)+m_{0}\right)}}{\ell(t)^{8}}, \\
\tilde{\rho}(x, t):=e^{s \tilde{\alpha}(x, t)}, \rho_{*}(t):=\exp \left(s \max _{x \in \bar{\Omega}} \tilde{\alpha}(x, t)\right), \xi_{*}(t):=\max _{x \in \bar{\Omega}} \tilde{\xi}(x, t) .
\end{array}\right.
$$

Then, the functions $\tilde{\xi}, \tilde{\rho}$ and $\rho_{*}$ are strictly positive and bounded in any set of the form $\bar{\Omega} \times[0, T-\delta]$ with $\delta>0$.

One has the following:

Lemma 2.3. There exists $\lambda_{0}>0$ such that, for each $\lambda \geq \lambda_{0}$, there exist $s_{0}$ and $C$ with the following property: for any $F \in L^{2}(Q)^{N}$, any $\varphi_{T} \in H$ and any $s \geq s_{0}$, the corresponding solution to (6) satisfies

$$
\begin{gathered}
\iint_{Q} \rho_{*}^{-2}\left(s \xi_{*}\right)^{-1}\left(\left|\varphi_{t}\right|^{2}+|\nabla \pi|^{2}\right) d x d t+\iint_{Q} \tilde{\rho}^{-2}(s \tilde{\xi})|\nabla \times \varphi|^{2} d x d t \\
\quad+\iint_{Q} \tilde{\rho}^{-2}\left((s \tilde{\xi})^{-1}|\Delta \varphi|^{2}+|\nabla \varphi|^{2}+(s \tilde{\xi})^{2}|\varphi|^{2}\right) d x d t \\
\leq C\left(\iint_{Q} \tilde{\rho}^{-2}|F|^{2} d x d t+\iint_{\omega \times(0, T)} \tilde{\rho}^{-2}(s \tilde{\xi})^{3}|\varphi|^{2} d x d t\right) .
\end{gathered}
$$

Proof - The proof is not difficult and relies on 12 and the usual energy estimates for $\varphi$. Since the main ideas are well known and can be found in many papers, we will only give a sketch.

Let us take $\lambda \geq \lambda_{0}$ and $s \geq s_{0}$ and let us set

$$
\begin{aligned}
\Gamma(\varphi, \pi ; a, b) & :=\iint_{\Omega \times(a, b)} \rho_{*}^{-2}\left(s \xi_{*}\right)^{-1}\left(\left|\varphi_{t}\right|^{2}+|\nabla \pi|^{2}\right) d x d t \\
& +\iint_{\Omega \times(a, b)} \tilde{\rho}^{-2}(s \tilde{\xi})|\nabla \times \varphi|^{2} d x d t \\
& +\iint_{\Omega \times(a, b)} \tilde{\rho}^{-2}\left((s \tilde{\xi})^{-1}|\Delta \varphi|^{2}+|\nabla \varphi|^{2}+(s \tilde{\xi})^{2}|\varphi|^{2}\right) d x d t
\end{aligned}
$$

and

$$
S(\varphi ; F):=\iint_{Q} \tilde{\rho}^{-2}|F|^{2} d x d t+\iint_{\omega \times(0, T)} \tilde{\rho}^{-2}(s \tilde{\xi})^{3}|\varphi|^{2} d x d t .
$$

Then $\Gamma(\varphi, \pi ; 0, T)=\Gamma(\varphi, \pi ; 0, T / 2)+\Gamma(\varphi, \pi ; T / 2, T)$ and, from Lemma 2.2 we clearly have

$$
\Gamma(\varphi, \pi ; T / 2, T) \leq C S(\varphi ; F) .
$$

On the other hand,

$$
\Gamma(\varphi, \pi ; 0, T / 2) \leq C \int_{0}^{T / 2}\left(\left\|\varphi_{t}\right\|^{2}+\|\Delta \varphi\|^{2}+\|\nabla \varphi\|^{2}+\|\varphi\|^{2}\right) d t .
$$


Let us check that this integral can be bounded similarly.

From (6), it is readily seen that

$$
-\frac{1}{2} \frac{d}{d t}\|\varphi\|^{2}+\nu\|\nabla \varphi\|^{2}=(F, \varphi) \leq \frac{\nu}{2}\|\nabla \varphi\|^{2}+C\|F\|^{2} \text { in }(0, T),
$$

whence

$$
\left\|\varphi\left(\cdot, t_{1}\right)\right\|^{2}+\int_{t_{1}}^{t_{2}}\|\nabla \varphi(\cdot, s)\|^{2} d s \leq\left\|\varphi\left(\cdot, t_{2}\right)\right\|^{2}+C \int_{t_{1}}^{t_{2}}\|F(\cdot, s)\|^{2} d s
$$

for all $0 \leq t_{1} \leq t_{2} \leq T$. In particular, taking $t_{1} \in[0, T / 2]$ and $t_{2} \in[T / 2,3 T / 4]$ and integrating with respect to $t_{1}$ and then with respect to $t_{2}$, we see that

$$
\int_{0}^{T / 2}\|\varphi(\cdot, t)\|^{2} d t \leq C\left(\int_{T / 2}^{3 T / 4}\|\varphi(\cdot, s)\|^{2} d s+\int_{0}^{3 T / 4}\|F(\cdot, s)\|^{2} d s\right) \leq C S(\varphi, F) .
$$

Also,

$$
\int_{0}^{T / 2}\|\nabla \varphi(\cdot, t)\|^{2} d t \leq\left\|\varphi\left(\cdot, t_{2}\right)\right\|^{2}+C \int_{0}^{3 T / 4}\|F(\cdot, s)\|^{2} d s
$$

for all $t_{2} \in[T / 2,3 T / 4]$ and, integrating with respect to $t_{2}$ in this interval, we deduce an estimate of the integral of $\|\nabla \varphi\|^{2}$ in $(0, T / 2)$ :

$$
\int_{0}^{T / 2}\|\nabla \varphi(\cdot, t)\|^{2} d t \leq C\left(\int_{T / 2}^{3 T / 4}\|\varphi(\cdot, s)\|^{2} d s+\int_{0}^{3 T / 4}\|F(\cdot, s)\|^{2} d s\right) \leq C S(\varphi, F) .
$$

A similar argument holds for the integral of $\|\Delta \varphi\|^{2}$. Indeed, one has

$$
-\frac{1}{2} \frac{d}{d t}\|\nabla \varphi\|^{2}+\nu\|\Delta \varphi\|^{2}=(F,-\Delta \varphi) \leq \frac{\nu}{2}\|\Delta \varphi\|^{2}+C\|F\|^{2} \text { in }(0, T) .
$$

Arguing as before, we see that

$$
\int_{0}^{T / 2}\|\Delta \varphi(\cdot, t)\|^{2} d t \leq\left\|\nabla \varphi\left(\cdot, t_{2}\right)\right\|^{2}+C \int_{0}^{3 T / 4}\|F(\cdot, s)\|^{2} d s
$$

for all $t_{2} \in[T / 2,3 T / 4]$ and, after integration with respect to $t_{2}$, we arrive at the estimate

$$
\int_{0}^{T / 2}\|\Delta \varphi(\cdot, t)\|^{2} d t \leq C\left(\int_{T / 2}^{3 T / 4}\|\nabla \varphi(\cdot, s)\|^{2} d s+\int_{0}^{3 T / 4}\|F(\cdot, s)\|^{2} d s\right) \leq C S(\varphi, F) .
$$

Finally, using that $\left\|\varphi_{t}\right\|^{2}=(\nu \Delta \varphi+F, \varphi)$, we find that

$$
\int_{0}^{T / 2}\left\|\varphi_{t}\right\|^{2} d t \leq C S(\varphi, F)
$$


As a consequence, $\Gamma(\varphi, \pi ; 0, T / 2) \leq C S(\varphi ; F)$ and the proof is done.

From now on, we will fix $\lambda$ and $s$ as in this lemma and we will consider the corresponding functions $\alpha, \tilde{\alpha}, \rho$, etc. in 10 and $(14)$. Also, we will introduce the function

$$
\tilde{\eta}:=\tilde{\rho} \tilde{\xi}^{-1} .
$$

An immediate consequence of Lemma 2.3 is the following:

Corollary 2.1. There exist positive constants $\lambda, s$ and $C$ only depending on $\Omega$, $\omega, T$ and $\nu$ such that, for any $F \in L^{2}(Q)^{N}$ and any $\varphi_{T} \in H$, the corresponding solution to (6) satisfies

$$
\iint_{Q} \tilde{\eta}^{-2}|\varphi|^{2} d x d t \leq C\left(\iint_{Q} \tilde{\rho}^{-2}|F|^{2} d x d t+\iint_{\omega \times(0, T)} \tilde{\eta}^{-2}|\varphi|^{2} d x d t\right) .
$$

\subsection{The null controllability of (5)}

We are dealing here with the Stokes system (5).

We will need some specific conditions on $f$ and $y_{0}$ to get the null controllability of (5); we will use the arguments in [14.

The following result holds:

Proposition 2.1. Let us assume that

$$
y_{0} \in H \quad \text { and } \tilde{\eta} f \in L^{2}(Q)^{N} .
$$

Then, we can find a control-state pair $(v, y)$ for (5) satisfying

$$
v \in L^{2}(\omega \times(0, T))^{N}, \quad y \in L^{2}(0, T ; V) \cap C^{0}([0, T] ; H),
$$

such that

$$
\iint_{Q} \tilde{\rho}^{2}|y|^{2} d x d t+\iint_{\omega \times(0, T)} \tilde{\eta}^{2}|v|^{2} d x d t<+\infty .
$$

In particular, one has $y(\cdot, T)=0$.

The proof relies on (15) and can be easily obtained arguing as in 20; see also [14. The key idea is to consider the extremal problem

$$
\left\{\begin{array}{l}
\text { Minimize } \iint_{Q} \tilde{\rho}^{2}|y|^{2} d x d t+\iint_{\omega \times(0, T)} \tilde{\eta}^{2}|v|^{2} d x d t, \\
\text { Subject to } v \in L^{2}(\omega \times(0, T))^{N},(y, p, v) \text { satisfies (5). }
\end{array}\right.
$$

Thanks to 15 , there exists exactly one solution to 20 and this solution is the desired control-state pair. 
It is also true that, in a certain sense, the solution to 20 depends continuously on the data $\left(f, y_{0}\right)$. In particular, if the $\left(f_{n}, y_{0 n}\right)$ satisfy

$$
\left\|y_{0 n}-y_{0}\right\| \rightarrow 0 \text { and } \iint_{Q} \tilde{\eta}^{2}\left|f_{n}-f\right|^{2} d x d t \rightarrow 0
$$

then the control-state pairs $\left(v_{n}, y_{n}\right)$ associated by Proposition 2.1 satisfy

$$
\iint_{Q} \tilde{\rho}^{2}\left|y_{n}-y\right|^{2} d x d t \rightarrow 0 \text { and } \iint_{\omega \times(0, T)} \tilde{\eta}^{2}\left|v_{n}-v\right|^{2} d x d t \rightarrow 0,
$$

where $(v, y)$ is the pair that corresponds to $\left(f, y_{0}\right)$. This will be used below, in Section 4 .

For brevity we omit the details, that can be found in [14].

\subsection{Some additional estimates}

The state found in Proposition 2.1 satisfies some additional properties, that will be needed below, in Section 3 . More precisely, we will show in this Section that not only $y$, but also $\nabla y, y_{t}$ and $\Delta y$ belong to weighted $L^{2}$ spaces.

Let us introduce the spatially homogeneous weights

$$
\hat{\rho}(t):=\exp \left(s \min _{x \in \bar{\Omega}} \tilde{\alpha}(x, t)\right), \quad \zeta(t):=\hat{\rho}(t) \ell(t)^{12}, \quad \gamma(t):=\hat{\rho}(t) \ell(t)^{33 / 2} .
$$

Then the following holds:

Proposition 2.2. Let the hypotheses in Proposition 2.1 be satisfied and let $(y, p, v)$ satisfy (5) and (19). Then

$$
\begin{gathered}
\max _{[0, T]} \int_{\Omega} \zeta^{2}|y|^{2} d x+\iint_{Q} \zeta^{2}|\nabla y|^{2} d x d t \leq C\left(\left\|y_{0}\right\|^{2}+\iint_{Q} \tilde{\rho}^{2}|y|^{2} d x d t\right. \\
\left.+\iint_{\omega \times(0, T)} \tilde{\eta}^{2}|v|^{2} d x d t+\iint_{Q} \tilde{\eta}^{2}|f|^{2} d x d t\right)
\end{gathered}
$$

Proof - Let us multiply the PDE in (5) by $\zeta^{2} y$ and let us integrate in $\Omega$. We obtain:

$$
\int_{\Omega} \zeta^{2}\left(y_{t}-\nu \Delta y+\nabla p\right) \cdot y d x=\int_{\Omega} \zeta^{2}\left(v 1_{\omega}+f\right) \cdot y d x .
$$

In view of the definitions of $\tilde{\rho}, \tilde{\eta}$ and $\zeta$ respectively in (14), 16) and 22, , one has:

$$
\zeta \leq C \tilde{\eta}, \quad\left|\zeta \zeta_{t}\right| \leq C \tilde{\rho}^{2} .
$$


Consequently, the following estimates hold:

$$
\begin{gathered}
\int_{\Omega} \zeta^{2} v 1_{\omega} \cdot y d x \leq C\left(\int_{\omega} \tilde{\eta}^{2}|v|^{2} d x\right)^{1 / 2}\left(\int_{\Omega} \zeta^{4} \tilde{\eta}^{-2}|y|^{2} d x\right)^{1 / 2} \\
\leq \frac{1}{2} \int_{\omega} \tilde{\eta}^{2}|v|^{2} d x+C \int_{\Omega} \tilde{\rho}^{2}|y|^{2} d x \\
\int_{\Omega} \zeta^{2} f \cdot y d x \leq \frac{1}{2} \int_{\Omega} \tilde{\eta}^{2}|f|^{2} d x+C \int_{\Omega} \tilde{\rho}^{2}|y|^{2} d x \\
\int_{\Omega} \zeta^{2} y_{t} \cdot y d x=\frac{1}{2} \frac{d}{d t} \int_{\Omega} \zeta^{2}|y|^{2} d x-\int_{\Omega} \zeta \zeta_{t}|y|^{2} d x \\
\geq \frac{1}{2} \frac{d}{d t} \int_{\Omega} \zeta^{2}|y|^{2} d x-C \int_{\Omega} \tilde{\rho}^{2}|y|^{2} d x
\end{gathered}
$$

On the other hand,

$$
\int_{\Omega} \zeta^{2} \nabla p \cdot y d x d t=0 \text { and } \int_{\Omega} \zeta^{2}(-\Delta y) \cdot y d x=\int_{\Omega} \zeta^{2}|\nabla y|^{2} d x .
$$

Accordingly, one has:

$$
\begin{aligned}
& \frac{1}{2} \frac{d}{d t} \int_{\Omega} \zeta^{2}|y|^{2} d x+\nu \int_{\Omega} \zeta^{2}|\nabla y|^{2} d x \\
& \quad \leq C\left(\int_{\Omega} \tilde{\eta}^{2}|f|^{2} d x+\int_{\omega} \tilde{\eta}^{2}|v|^{2} d x+\int_{\Omega} \tilde{\rho}^{2}|y|^{2} d x\right) .
\end{aligned}
$$

Now, integrating in time, the estimate 23 is easily found.

Proposition 2.3. Let the hypotheses in Proposition 2.1 be satisfied and let $(y, p, v)$ satisfy (5) and (19). Let us assume that

$$
y_{0} \in V \text {. }
$$

Then one has

$$
\begin{aligned}
& \max _{[0, T]} \int_{\Omega} \gamma^{2}|\nabla y|^{2} d x+\iint_{Q} \gamma^{2}\left(\left|y_{t}\right|^{2}+|\Delta y|^{2}\right) d x d t \leq C\left(\left\|y_{0}\right\|_{H_{0}^{1}}^{2}\right. \\
& \left.+\iint_{Q} \tilde{\rho}^{2}|y|^{2} d x d t+\iint_{\omega \times(0, T)} \tilde{\eta}^{2}|v|^{2} d x d t+\iint_{Q} \tilde{\eta}^{2}|f|^{2} d x d t\right) .
\end{aligned}
$$

Proof - Notice that, under the assumption (24), the solution $(y, p)$ to (5) satisfies

$$
y \in L^{2}(0, T ; D(A)) \cap C^{0}([0, T] ; V), \quad y_{t} \in L^{2}(0, T ; H),
$$

where $A: D(A) \mapsto H$ is the Stokes operator. 
Let us multiply the PDE in (5) by $\gamma^{2} y_{t}$ and let us integrate in $\Omega$. The following holds:

$$
\int_{\Omega} \gamma^{2}\left(y_{t}-\nu \Delta y+\nabla p\right) \cdot y_{t} d x=\int_{\Omega} \gamma^{2}\left(v 1_{\omega}+f\right) \cdot y_{t} d x .
$$

From the definition of $\gamma$, we see that

$$
\gamma \leq C \zeta \leq C \tilde{\eta}, \quad\left|\gamma \gamma_{t}\right| \leq C \zeta^{2} .
$$

Consequently, for any small $\epsilon>0$, we find that

$$
\begin{gathered}
\int_{\Omega} \gamma^{2} v 1_{\omega} \cdot y_{t} d x \leq \epsilon \int_{\Omega} \gamma^{2}\left|y_{t}\right|^{2} d x+C_{\epsilon} \int_{\omega} \tilde{\eta}^{2}|v|^{2} d x \\
\int_{\Omega} \gamma^{2} f \cdot y_{t} d x \leq \epsilon \int_{\Omega} \gamma^{2}\left|y_{t}\right|^{2} d x+C_{\epsilon} \int_{\Omega} \tilde{\eta}^{2}|f|^{2} d x
\end{gathered}
$$

and, also,

$$
\begin{gathered}
-\int_{\Omega} \gamma^{2} \Delta y \cdot y_{t} d x=\frac{1}{2} \frac{d}{d t} \int_{\Omega} \gamma^{2}|\nabla y|^{2} d x-\int_{\Omega} \gamma \gamma_{t}|\nabla y|^{2} d x \\
\geq \frac{1}{2} \frac{d}{d t} \int_{\Omega} \gamma^{2}|\nabla y|^{2} d x-C \int_{\Omega} \zeta^{2}|\nabla y|^{2} d x
\end{gathered}
$$

On the other hand, the integral involving the pressure vanishes. Therefore, the following is found integrating in time:

$$
\begin{gathered}
\iint_{Q} \gamma^{2}\left|y_{t}\right|^{2} d x+\max _{[0, T]} \int_{\Omega} \gamma^{2}|\nabla y|^{2} d x \leq C\left(\left\|y_{0}\right\|_{H_{0}^{1}(\Omega)}^{2}\right. \\
\left.+\iint_{Q} \zeta^{2}|\nabla y|^{2} d x d t+\iint_{\omega \times(0, T)} \tilde{\eta}^{2}|v|^{2} d x d t+\iint_{Q} \tilde{\eta}^{2}|f|^{2} d x d t\right) .
\end{gathered}
$$

This furnishes the estimates of $\gamma^{2}\left|y_{t}\right|^{2}$ and $\gamma^{2}|\nabla y|^{2}$ in (25).

In order to estimate the weighted integral of $|\Delta y|^{2}$, let us multiply the PDE in (5) by $\gamma^{2} A y$ (recall that $A$ is the Stokes operator). After integration in $\Omega$, we have:

$$
\int_{\Omega} \gamma^{2}\left(y_{t}-\nu \Delta y+\nabla p\right) \cdot A y d x=\int_{\Omega} \gamma^{2}\left(v 1_{\omega}+f\right) \cdot A y d x .
$$

Observe that

$$
\begin{gathered}
\int_{\Omega} \gamma^{2} v 1_{\omega} \cdot A y d x \leq \epsilon \int_{\Omega} \gamma^{2}|\Delta y|^{2} d x+C_{\epsilon} \int_{\omega} \tilde{\eta}^{2}|v|^{2} d x \\
\int_{\Omega} \gamma^{2} f \cdot A y d x \leq \epsilon \int_{\Omega} \gamma^{2}|\Delta y|^{2} d x+C_{\epsilon} \int_{\Omega} \tilde{\eta}^{2}|f|^{2} d x \\
\int_{\Omega} \gamma^{2} y_{t} \cdot A y d x=\int_{\Omega} \gamma^{2} y_{t} \cdot(-\Delta y) d x \\
\leq \epsilon \int_{\Omega} \gamma^{2}|\Delta y|^{2} d x+C_{\epsilon} \int_{\Omega} \gamma^{2}\left|y_{t}\right|^{2} d x
\end{gathered}
$$


for any small $\epsilon>0$,

$$
\int_{\Omega} \gamma^{2} \nabla p \cdot A y d x=0 \text { and } \int_{\Omega} \gamma^{2}(-\Delta y) \cdot A y d x=\int_{\Omega} \gamma^{2}|\Delta y|^{2} d x .
$$

Integrating in time, we see now that

$$
\iint_{Q} \gamma^{2}|\Delta y|^{2} d x d t \leq C\left(\iint_{\omega \times(0, T)} \tilde{\eta}^{2}|v|^{2} d x d t+\iint_{Q} \tilde{\eta}^{2}|f|^{2} d x d t+\iint_{Q} \gamma^{2}\left|y_{t}\right|^{2} d x d t\right) .
$$

From $(26)$ and this last inequality, we obtain 25 .

\section{The proof of Theorem 1.1}

In this Section, we will prove the local null controllability of the system (1). Thus, let us set

$$
L y:=y_{t}-\nu \Delta y
$$

and let us introduce the space

$$
\begin{aligned}
E_{N}:= & \left\{(y, p, v): \tilde{\rho} y \in L^{2}(Q)^{N}, \tilde{\eta} v \in L^{2}(\omega \times(0, T))^{N},\right. \\
& y \in L^{2}(0, T ; D(A)), p \in L^{2}\left(0, T ; H^{1}(\Omega)\right), \int_{\Omega} p d x=0 \text { a.e., } \\
& \left.\tilde{\eta}\left(L y+\nabla p-v 1_{\omega}\right) \in L^{2}(Q)^{N}\right\} .
\end{aligned}
$$

It is clear that $E_{N}$ is a Hilbert space for the norm $\|\cdot\|_{E_{N}}$, where

$$
\begin{aligned}
\|(y, p, v)\|_{E_{N}} & :=\left(\|\tilde{\rho} y\|_{L^{2}(Q)}^{2}+\|\tilde{\eta} v\|_{L^{2}(\omega \times(0, T))}^{2}\right. \\
& +\|y\|_{L^{2}(0, T ; D(A))}^{2}+\|p\|_{L^{2}\left(0, T ; H^{1}(\Omega)\right)}^{2} \\
& \left.+\left\|\tilde{\eta}\left(L y+\nabla p-v 1_{\omega}\right)\right\|_{L^{2}(Q)}^{2}\right)^{1 / 2} .
\end{aligned}
$$

Notice that, if $(y, p, v) \in E_{N}$, then $y_{t} \in L^{2}(Q)^{N}$, whence $y:[0, T] \mapsto V$ is (absolutely) continuous and, in particular, we have $y(\cdot, 0) \in V$ and

$$
\|y(\cdot, 0)\|_{V} \leq C\|(y, p, v)\|_{E_{N}} \quad \forall(y, p, v) \in E_{N} .
$$

Furthermore, in view of Propositions 2.2 and 2.3 , one also has $\zeta y \in L^{2}(0, T ; V) \cap$ $L^{\infty}(0, T ; H)$ and $\gamma y \in L^{2}(0, T ; D(A)) \cap L^{\infty}(0, T ; V)$, with norms bounded again by $C\|(y, p, v)\|_{E_{N}}$.

Let $L^{2}\left(\tilde{\eta}^{2} ; Q\right)$ be the Hilbert space formed by the measurable functions $w=$ $w(x, t)$ such that $\tilde{\eta} w \in L^{2}(Q)$, i.e.

$$
\|w\|_{L^{2}\left(\tilde{\eta}^{2} ; Q\right)}^{2}:=\iint_{Q} \tilde{\eta}^{2}|w|^{2} d x d t<+\infty .
$$


Let us introduce the Hilbert space $Z_{N}:=L^{2}\left(\tilde{\eta}^{2} ; Q\right)^{N} \times V$ and the mapping $\mathcal{A}: E_{N} \mapsto Z_{N}$, given by

$$
\mathcal{A}(y, p, v):=\left(y_{t}-\nabla \cdot\left(\left(\nu_{0}+\nu_{1}\left(\|D y\|^{2}\right)\right) D y\right)+(y \cdot \nabla) y+\nabla p-v 1_{\omega}, y(\cdot, 0)\right) .
$$

Notice that, in this definition, $\nabla \cdot\left(\left(\nu_{0}+\nu_{1}\left(\|D y\|^{2}\right)\right) D y\right)$ can be rewritten in the form $\left(\nu_{0}+\nu_{1}\left(\|D y\|^{2}\right)\right) \Delta y$.

Our aim is to apply the following version of Liusternik's Inverse Function Theorem in infinite dimensional spaces, that can be found for instance in [1. In the following statement, $B_{r}(0)$ and $B_{\epsilon}\left(\zeta_{0}\right)$ are open balls, respectively of radius $r$ and $\epsilon$.

Theorem 3.1. Let $Y$ and $Z$ be Banach spaces and let $H: B_{r}(0) \subset Y \mapsto Z$ be a $C^{1}$ mapping. Let us assume that the derivative $H^{\prime}(0): Y \mapsto Z$ is onto and let us set $\zeta_{0}=H(0)$. Then there exist $\epsilon>0$, a mapping $W: B_{\epsilon}\left(\zeta_{0}\right) \subset Z \mapsto Y$ and a constant $K>0$ satisfying:

$$
\left\{\begin{array}{l}
W(z) \in B_{r}(0) \text { and } H(W(z))=z \quad \forall z \in B_{\epsilon}\left(\zeta_{0}\right) \\
\|W(z)\|_{Y} \leq K\|z-H(0)\|_{Z} \quad \forall z \in B_{\epsilon}\left(\zeta_{0}\right)
\end{array}\right.
$$

Notice that, in this theorem, $W$ is the inverse-to-the-right of $H$.

In order to show that Theorem 3.1 can be applied in this setting, we will use several lemmas.

Lemma 3.1. Let $\mathcal{A}: E_{N} \mapsto Z_{N}$ be the mapping defined by (29). Then, $\mathcal{A}$ is well defined and continuous.

Proof - First, note that, in view of (11) and 22, we have

$$
\tilde{\eta}^{2} \leq C \zeta \gamma^{3} \leq C \gamma^{6} .
$$

Let us denote by $\mathcal{A}_{i}$ the components of $\mathcal{A}$ for $1 \leq i \leq N+1$. Then $\mathcal{A}_{i}(y, p, v) \in L^{2}\left(\tilde{\eta}^{2} ; Q\right)$ for $1 \leq i \leq N$ and $\mathcal{A}_{N+1}(y, p, v) \in V$ for every $(y, p, v) \in$ $E_{N}$.

Indeed, one has:

$$
\begin{aligned}
\iint_{Q} \tilde{\eta}^{2} & \sum_{i=1}^{N}\left|\mathcal{A}_{i}(y, p, v)\right|^{2} d x d t \\
\leq & 3 \iint_{Q} \tilde{\eta}^{2}\left|y_{t}-\nu \Delta y+\nabla p-v 1_{\omega}\right|^{2} d x d t \\
& +3 \iint_{Q} \tilde{\eta}^{2}\left|\nabla \cdot\left(\left(\nu_{1}(0)-\nu_{1}\left(\|D y\|^{2}\right)\right) D y\right)\right|^{2} d x d t \\
& +3 \iint_{Q} \tilde{\eta}^{2}|(y \cdot \nabla) y|^{2} d x d t \\
& :=3 M_{1}+3 M_{2}+3 M_{3} .
\end{aligned}
$$


From the definition of $E_{N}$, we have:

$$
M_{1} \leq\|(y, p, v)\|_{E_{N}}^{2} .
$$

On the other hand, taking into account that $\|\nabla w\|_{L^{3}} \leq C\|\nabla w\|^{1 / 2}\|\Delta w\|^{1 / 2}$ and

$$
\|(w \cdot \nabla) w\|^{2} \leq C\|w\|_{L^{6}}^{2}\|\nabla w\|_{L^{3}}^{2} \leq C\|\nabla w\|^{3}\|\Delta w\|
$$

for all $w \in D(A)$, we have:

$$
\begin{aligned}
M_{3} & \leq C \int_{0}^{T} \zeta \gamma^{3}\|(y(\cdot, t) \cdot \nabla) y(\cdot, t)\|^{2} d t \\
& \leq C \int_{0}^{T} \zeta \gamma^{3}\|\nabla y(\cdot, t)\|^{3}\|\Delta y(\cdot, t)\| d t \\
& \leq C\|\zeta y\|_{L^{2}(0, T ; V)}\|\gamma y\|_{L^{\infty}(0, T ; V)}^{2}\|\gamma y\|_{L^{2}(0, T ; D(A))} \leq C\|(y, p, v)\|_{E_{N}}^{4} .
\end{aligned}
$$

Finally, from Proposition 2.3 , the hypotheses on $\nu_{1}$ and $(30)$, we deduce that

$$
\begin{aligned}
M_{2} & =\iint_{Q} \tilde{\eta}^{2}\left|\nu_{1}(0)-\nu_{1}\left(\|D y\|^{2}\right)\right|^{2}|\Delta y|^{2} d x d t \\
& \leq C \iint_{Q} \gamma^{6}\|\nabla y\|^{4}|\Delta y|^{2} d x d t \\
& \leq C\left(\sup _{[0, T]} \gamma^{2}\|\nabla y\|^{2}\right)^{2}\left(\iint_{Q} \gamma^{2}|\Delta y|^{2} d x d t\right) \\
& \leq C\|(y, p, v)\|_{E_{N}}^{6} .
\end{aligned}
$$

Consequently, $\mathcal{A}$ takes values in $Z_{N}$.

That the mapping $\mathcal{A}$ is continuous is easy to prove using similar arguments. Thus, let us assume that $\left(y_{n}, p_{n}, v_{n}\right) \rightarrow(y, p, v)$ in $E_{N}$ and let us see that $\mathcal{A}\left(y_{n}, p_{n}, v_{n}\right) \rightarrow \mathcal{A}(y, p, v)$ in $Z_{N}$.

Obviously, $y_{n}(\cdot, 0) \rightarrow y(\cdot, 0)$ in $V$. Moreover,

$$
\begin{aligned}
& \iint_{Q} \tilde{\eta}^{2} \sum_{i=1}^{N}\left|\mathcal{A}_{i}\left(y_{n}, p_{n}, v_{n}\right)-\mathcal{A}_{i}(y, p, v)\right|^{2} d x d t \\
& \quad \leq 3 \iint_{Q} \tilde{\eta}^{2}\left|\left(L y_{n}+\nabla p_{n}-v_{n} 1_{\omega}\right)-\left(L y+\nabla p-v 1_{\omega}\right)\right|^{2} d x d t \\
& \quad+3 \iint_{Q} \tilde{\eta}^{2}\left|\left(\nu_{1}(0)-\nu_{1}\left(\left\|D y_{n}\right\|^{2}\right)\right) \Delta y_{n}-\left(\nu_{1}(0)-\nu_{1}\left(\|D y\|^{2}\right)\right) \Delta y\right|^{2} d x d t \\
& \quad+3 \iint_{Q} \tilde{\eta}^{2}\left|\left(y_{n} \cdot \nabla\right) y_{n}-(y \cdot \nabla) y\right|^{2} d x d t \\
& \quad:=3 Z_{1, n}+3 Z_{2, n}+3 Z_{3, n} .
\end{aligned}
$$

Since $Z_{1, n} \leq\left\|\left(y_{n}, p_{n}, v_{n}\right)-(y, p, v)\right\|_{E_{N}}^{2}$, one has $Z_{1, n} \rightarrow 0$. 
On the other hand,

$$
\begin{aligned}
Z_{2, n} \leq & 2 \iint_{Q} \tilde{\eta}^{2}\left|\nu_{1}(0)-\nu_{1}\left(\left\|D y_{n}\right\|^{2}\right)\right|^{2}\left|\Delta\left(y_{n}-y\right)\right|^{2} d x d t \\
& +2 \iint_{Q} \tilde{\eta}^{2}\left|\nu_{1}\left(\left\|D y_{n}\right\|^{2}\right)-\nu_{1}\left(\|D y\|^{2}\right)\right|^{2}|\Delta y|^{2} d x d t \\
\leq & C \iint_{Q} \gamma^{6}\left\|D y_{n}\right\|^{2}\left|\Delta\left(y_{n}-y\right)\right|^{2} d x d t \\
& +C \iint_{Q} \gamma^{6}\left\|D\left(y_{n}-y\right)\right\|^{2}\left\|D\left(y_{n}+y\right)\right\|^{2}|\Delta y|^{2} d x d t \\
\leq & C\left(\left\|\left(y_{n}, p_{n}, v_{n}\right)\right\|_{E_{N}}^{4}+\|(y, p, v)\|_{E_{N}}^{4}\right)\left\|\left(y_{n}, p_{n}, v_{n}\right)-(y, p, v)\right\|_{E_{N}}^{2},
\end{aligned}
$$

whence we also have $Z_{2, n} \rightarrow 0$.

Finally,

$$
\begin{aligned}
Z_{3, n} \leq & 2 \iint_{Q} \tilde{\eta}^{2}\left[\left|\left(\left(y_{n}-y\right) \cdot \nabla\right) y_{n}\right|^{2}+\left|(y \cdot \nabla)\left(y_{n}-y\right)\right|^{2}\right] d x d t \\
\leq & C \int_{0}^{T} \zeta \gamma^{3}\left[\left\|y_{n}-y\right\|_{L^{6}}^{2}\left\|\nabla y_{n}\right\|_{L^{3}}^{2}+\|y\|_{L^{6}}^{2}\left\|\nabla\left(y_{n}-y\right)\right\|_{L^{3}}^{2}\right] d t \\
\leq & C \int_{0}^{T} \zeta \gamma^{3}\left\|\nabla\left(y_{n}-y\right)\right\|^{2}\left\|\nabla y_{n}\right\|\left\|\Delta y_{n}\right\| d t \\
& +C \int_{0}^{T} \zeta \gamma^{3}\|\nabla y\|^{2}\left\|\nabla\left(y_{n}-y\right)\right\|\left\|\Delta\left(y_{n}-y\right)\right\| d t \\
\leq & C\left(\left\|\left(y_{n}, p_{n}, v_{n}\right)\right\|_{E_{N}}^{2}+\|(y, p, v)\|_{E_{N}}^{2}\right)\left\|\left(y_{n}, p_{n}, v_{n}\right)-(y, p, v)\right\|_{E_{N}}^{2}
\end{aligned}
$$

and we see that $Z_{3, n} \rightarrow 0$.

This shows that $\mathcal{A}\left(y_{n}, p_{n}, v_{n}\right) \rightarrow \mathcal{A}(y, p, v)$ in $Z_{N}$ and ends the proof.

Lemma 3.2. The mapping $\mathcal{A}: E_{N} \mapsto Z_{N}$ is continuously differentiable.

Proof - We will present the proof when $N=3$. The proof for $N=2$ is similar.

Let us first prove that $\mathcal{A}$ is G-differentiable at any $(y, p, v) \in E_{N}$ and let us compute the G-derivative $\mathcal{A}^{\prime}(x, y, z)$.

Thus, let us fix $(y, p, v)$ in $E_{3}$ and let us take $\left(y^{\prime}, p^{\prime}, v^{\prime}\right) \in E_{3}$ and $\sigma>0$. For simplicity, we will use the notation

$$
\begin{aligned}
& \nu_{1, \sigma}:=\nu_{1}\left(\left\|D\left(y+\sigma y^{\prime}\right)\right\|^{2}\right), \quad \nu_{1, \sigma}^{\prime}:=\nu_{1}^{\prime}\left(\left\|D\left(y+\sigma y^{\prime}\right)\right\|^{2}\right), \\
& \tilde{\nu}_{1, n}:=\nu_{1}\left(\left\|D y_{n}\right\|^{2}\right), \quad \tilde{\nu}_{1, n}^{\prime}:=\nu_{1}^{\prime}\left(\left\|D y_{n}\right\|^{2}\right) .
\end{aligned}
$$

We have:

$$
\begin{aligned}
& \frac{1}{\sigma}\left[\mathcal{A}_{i}\left((y, p, v)+\sigma\left(y^{\prime}, p^{\prime}, v^{\prime}\right)\right)-\mathcal{A}_{i}(y, p, v)\right] \\
& \quad=y_{i t}^{\prime}-\left(\nu_{0}+\nu_{1, \sigma}\right) \Delta y_{i}^{\prime}-\frac{1}{\sigma}\left(\nu_{1, \sigma}-\nu_{1,0}\right) \Delta y_{i}+\frac{\partial p^{\prime}}{\partial x_{i}}-v_{i}^{\prime} 1_{\omega} \\
& \quad+y^{\prime} \cdot \nabla y_{i}+y \cdot \nabla y_{i}^{\prime}+\sigma y^{\prime} \cdot \nabla y_{i}^{\prime},
\end{aligned}
$$


for $1 \leq i \leq 3$.

Let us introduce the linear mapping $D \mathcal{A}: E_{3} \mapsto Z_{3}$, with

$$
\begin{gathered}
D \mathcal{A}(y, p, v)=D \mathcal{A}=\left(D \mathcal{A}_{1}, D \mathcal{A}_{2}, D \mathcal{A}_{3}, D \mathcal{A}_{4}\right) \\
D \mathcal{A}_{i}\left(y^{\prime}, p^{\prime}, v^{\prime}\right)=y_{i t}^{\prime}-\left(\nu_{0}+\nu_{1,0}\right) \Delta y_{i}^{\prime}-2 \nu_{1,0}^{\prime}\left(\nabla y, \nabla y^{\prime}\right) \Delta y_{i}+\frac{\partial p^{\prime}}{\partial x_{i}} \\
-v_{i}^{\prime} 1_{\omega}+y^{\prime} \cdot \nabla y_{i}+y \cdot \nabla y_{i}^{\prime}
\end{gathered}
$$

for $1 \leq i \leq 3$ and

$$
D \mathcal{A}_{4}\left(y^{\prime}, p^{\prime}, v^{\prime}\right)=y^{\prime}(\cdot, 0)
$$

for all $\left(y^{\prime}, p^{\prime}, v^{\prime}\right) \in E_{3}$.

From the definition of the spaces $E_{3}$ and $Z_{3}$ and $(34)-(35)$, it becomes clear that $D \mathcal{A} \in \mathcal{L}\left(E_{3} ; Z_{3}\right)$. Furthermore,

$$
\frac{1}{\sigma}\left[\mathcal{A}_{i}\left((y, p, v)+\sigma\left(y^{\prime}, p^{\prime}, v^{\prime}\right)\right)-\mathcal{A}_{i}(y, p, v)\right] \rightarrow D \mathcal{A}_{i}\left(y^{\prime}, p^{\prime}, v^{\prime}\right)
$$

strongly in $L^{2}\left(\tilde{\eta}^{2} ; Q\right)$ for $1 \leq i \leq 3$ as $\sigma \rightarrow 0$. Indeed, we have:

$$
\begin{aligned}
& \left\|\frac{1}{\sigma}\left[\mathcal{A}_{i}\left((y, p, v)+\sigma\left(y^{\prime}, p^{\prime}, v^{\prime}\right)\right)-\mathcal{A}_{i}(y, p, v)\right]-D \mathcal{A}_{i}\left(y^{\prime}, p^{\prime}, v^{\prime}\right)\right\|_{L^{2}\left(\tilde{\eta}^{2} ; Q\right)} \\
& \quad \leq\left\|\left(\nu_{1, \sigma}-\nu_{1,0}\right) \Delta y_{i}^{\prime}\right\|_{L^{2}\left(\tilde{\eta}^{2} ; Q\right)} \\
& \quad+\left\|\left[\frac{1}{\sigma}\left(\nu_{1 \sigma}-\nu_{1,0}\right)-2 \nu_{1,0}^{\prime}\left(\nabla y, \nabla y^{\prime}\right)\right] \Delta y_{i}\right\|_{L^{2}\left(\tilde{\eta}^{2} ; Q\right)}+\left\|\sigma y^{\prime} \cdot \nabla y_{i}^{\prime}\right\|_{L^{2}\left(\tilde{\eta}^{2} ; Q\right)} \\
& \quad:=B_{1}+B_{2}+B_{3} .
\end{aligned}
$$

Let us check that the $B_{i}$ converge to zero as $\sigma \rightarrow 0$.

First, using Proposition 2.3 and taking into account that $\nu_{1} \in C_{b}^{1}(\mathbb{R})$, we get the following as $\sigma \rightarrow 0$ :

$$
\begin{aligned}
B_{1}^{2} & \leq \iint_{Q} \tilde{\eta}^{2}\left|\nu_{1, \sigma}-\nu_{1,0}\right|^{2}\left|\Delta y^{\prime}\right|^{2} d x d t \\
& \leq C \iint_{Q} \tilde{\eta}^{2}\left|\left\|\nabla\left(y+\sigma y^{\prime}\right)\right\|^{2}-\|\nabla y\|^{2}\right|^{2}\left|\Delta y^{\prime}\right|^{2} d x d t \\
& \leq C \sigma^{2} \sup _{[0, T]}\left(\gamma^{2}\left[\|\nabla y\|^{2}+\left\|\nabla y^{\prime}\right\|^{2}\right]\right)^{2} \iint_{Q} \gamma^{2}\left|\Delta y^{\prime}\right|^{2} d x d t \\
& \leq C \sigma^{2}\left(\|(y, p, v)\|_{E_{3}}^{4}+\left\|\left(y^{\prime}, p^{\prime}, v^{\prime}\right)\right\|_{E_{3}}^{4}\right)\left\|\left(y^{\prime}, p^{\prime}, v^{\prime}\right)\right\|_{E_{3}}^{2} \rightarrow 0 .
\end{aligned}
$$

Also,

$$
\begin{aligned}
B_{2}^{2} & \leq \iint_{Q} \tilde{\eta}^{2}\left|\nu_{1, r}^{\prime}-\nu_{1, \sigma}^{\prime}\right|^{2}\|\nabla y\|^{2}\left\|\nabla y^{\prime}\right\|^{2}\left|\Delta y^{\prime}\right|^{2} d x d t \\
& \leq C \sup _{[0, T]}\left(\gamma^{4}\|\nabla y\|^{2}\left\|\nabla y^{\prime}\right\|^{2}\right) \iint_{Q} \gamma^{2}\left|\nu_{1, r}^{\prime}-\nu_{1,0}^{\prime}\right|^{2}\left|\Delta y^{\prime}\right|^{2} d x d t,
\end{aligned}
$$


where $r=r(t)$ and $0<|r| \leq|\sigma|$. Using Proposition 2.3 and Lebesgue's Theorem, we easily find that $B_{2} \rightarrow 0$.

For $B_{3}$, the argument is very similar.

Taking into account the behaviour of $B_{1}, B_{2}$ and $B_{3}$, we see that (36) is true, whence we deduce that $\mathcal{A}$ is $G$-differentiable at any $(y, p, v) \in E_{3}$, with a $G$-derivative $\mathcal{A}^{\prime}(y, p, v)=D \mathcal{A}$.

Now, we shall prove that the mapping $(y, p, v) \mapsto \mathcal{A}^{\prime}(y, p, v)$ is continuous from $E_{3}$ into $\mathcal{L}\left(E_{3} ; Z_{3}\right)$. As a consequence, in view of classical results, we will have that $\mathcal{A}$ is not only G-differentiable but also F-differentiable and $C^{1}$ and we will get the desired result (see for instance Theorem 1-2, p. 21 in 29 ).

Thus, let us assume that $\left(y^{n}, p^{n}, v^{n}\right) \rightarrow(y, p, v)$ in $E_{3}$ and let us check that

$$
\left\|\left(D \mathcal{A}\left(y^{n}, p^{n}, v^{n}\right)-D \mathcal{A}(y, p, v)\right)\left(y^{\prime}, p^{\prime}, v^{\prime}\right)\right\|_{Z_{3}}^{2} \leq \epsilon_{n}\left\|\left(y^{\prime}, p^{\prime}, v^{\prime}\right)\right\|_{E_{3}}^{2}
$$

for all $(y, p, v) \in E_{3}$, for some $\epsilon_{n} \rightarrow 0$.

The following holds:

$$
\begin{aligned}
& \left.\left\|\left(D \mathcal{A}_{i}\left(y^{n}, p^{n}, v^{n}\right)-D \mathcal{A}_{i}(y, p, v)\right)\left(y^{\prime}, p^{\prime}, v^{\prime}\right)\right\|\right|_{L^{2}\left(\tilde{\eta}^{2} ; Q\right)} ^{2} \\
& \quad \leq 3 \iint_{Q} \tilde{\eta}^{2}\left|\tilde{\nu}_{1, n}-\nu_{1,0}\right|^{2}\left|\Delta y_{i}^{\prime}\right|^{2} d x d t \\
& \quad+12 \iint_{Q} \tilde{\eta}^{2}\left|\tilde{\nu}_{1, n}^{\prime}\left(\nabla y^{n}, \nabla y^{\prime}\right) \Delta y_{i}^{n}-\nu_{1,0}^{\prime}\left(\nabla y, \nabla y^{\prime}\right) \Delta y_{i}\right|^{2} d x d t \\
& \quad+3 \iint_{Q} \tilde{\eta}^{2}\left|y^{\prime} \cdot\left(\nabla y_{i}^{n}-\nabla y_{i}\right)+\left(y^{n}-y\right) \cdot \nabla y_{i}^{\prime}\right|^{2} d x d t \\
& \quad:=3 D_{1, n}+12 D_{2, n}+3 D_{3, n} .
\end{aligned}
$$

It is tedious (but in fact not difficult) to check that the $D_{i, n}$ can be bounded as in (39). For instance, using again Proposition 2.3 and the fact that $\nu_{1} \in$ $C_{b}^{1}(\mathbb{R})$, we have

$$
\begin{aligned}
D_{1, n} & \leq C \iint_{Q} \tilde{\eta}^{2}\left\|\nabla y^{n}-\nabla y\right\|^{2}\left(\left\|\nabla y^{n}\right\|^{2}+\|\nabla y\|\right)^{2}\left|\Delta y^{\prime}\right|^{2} d x d t \\
& \leq C \sup _{[0, T]}\left(\gamma^{4}\left\|\nabla\left(y^{n}-y\right)\right\|^{2}\left(\left\|\nabla y^{n}\right\|^{2}+\|\nabla y\|^{2}\right)\right) \iint_{Q} \gamma^{2}\left|\Delta y^{\prime}\right|^{2} d x d t \\
& \leq C \epsilon_{1, n}\left\|\left(y^{\prime}, p^{\prime}, v^{\prime}\right)\right\|_{E_{3}}^{2},
\end{aligned}
$$

where

$$
\epsilon_{1, n}:=\left\|\left(y^{n}-y, p^{n}-p, v^{n}-v\right)\right\|_{E_{3}}^{2}\left(\left\|\left(y^{n}, p^{n}, v^{n}\right)\right\|_{E_{3}}^{2}+\|(y, p, v)\|_{E_{3}}^{2}\right) .
$$

For the other $D_{i, n}$, similar arguments lead to the same conclusion. 
Thus,

$$
\begin{aligned}
D_{2, n} \leq & 3 \iint_{Q} \tilde{\eta}^{2}\left|\tilde{\nu}_{1, n}^{\prime}\right|^{2}\left\|\nabla y^{n}\right\|^{2}\left\|\nabla y^{\prime}\right\|^{2}\left|\Delta\left(y_{i}^{n}-y_{i}\right)\right|^{2} d x d t \\
& +3 \iint_{Q} \tilde{\eta}^{2}\left|\tilde{\nu}_{1, n}^{\prime}\right|^{2}\left\|\nabla\left(y^{n}-y\right)\right\|^{2}\left\|\nabla y^{\prime}\right\|^{2}\left|\Delta y_{i}\right|^{2} d x d t \\
& +3 \iint_{Q} \tilde{\eta}^{2}\left|\tilde{\nu}_{1, n}^{\prime}-\tilde{\nu}_{1,0}^{\prime}\right|^{2}\|\nabla y\|^{2}\left\|\nabla y^{\prime}\right\|^{2}\left|\Delta y_{i}\right|^{2} d x d t \\
\leq & C \epsilon_{2, n}\left\|\left(y^{\prime}, p^{\prime}, v^{\prime}\right)\right\|_{E_{3}}^{2},
\end{aligned}
$$

where

$$
\begin{aligned}
\epsilon_{2, n}:= & \left(\sup _{[0, T]} \gamma\left\|\nabla y^{n}\right\|\right)^{2} \iint_{Q} \gamma^{2}\left|\Delta\left(y_{i}^{n}-y_{i}\right)\right|^{2} d x d t \\
& +\left(\sup _{[0, T]} \gamma\left\|\nabla\left(y^{n}-y\right)\right\|\right)^{2} \iint_{Q} \gamma^{2}\left|\Delta y_{i}\right|^{2} d x d t \\
& +\left(\sup _{[0, T]} \gamma\|\nabla y\|\right)^{2} \iint_{Q} \gamma^{2}\left|\tilde{\nu}_{1, n}^{\prime}-\tilde{\nu}_{1,0}^{\prime}\right|^{2}\left|\Delta y_{i}\right|^{2} d x d t .
\end{aligned}
$$

Also, one has

$$
\begin{aligned}
D_{3, n} \leq & 2 \iint_{Q} \tilde{\eta}^{2}\left\|y^{\prime}\right\|_{L^{6}}^{2}\left\|\nabla\left(y_{i}^{n}-y_{i}\right)\right\|_{L^{3}}^{2} d x d t \\
& +2 \iint_{Q} \tilde{\eta}^{2}\left\|y^{n}-y\right\|_{L^{6}}^{2}\left\|\nabla y_{i}^{\prime}\right\|_{L^{3}}^{2} d x d t \\
\leq & C \int_{0}^{T} \zeta \gamma^{3}\left\|\nabla y^{\prime}\right\|^{2}\left\|\nabla\left(y_{i}^{n}-y_{i}\right)\right\|\left\|\Delta\left(y_{i}^{n}-y_{i}\right)\right\| d t \\
& +C \int_{0}^{T} \zeta \gamma^{3}\left\|\nabla\left(y^{n}-y\right)\right\|^{2}\left\|\nabla y_{i}^{\prime}\right\|\left\|\Delta y_{i}^{\prime}\right\| d t \\
\leq & C \epsilon_{3, n}\left\|\left(y^{\prime}, p^{\prime}, v^{\prime}\right)\right\|_{E_{3}}^{2},
\end{aligned}
$$

where

$$
\begin{aligned}
\epsilon_{3, n}:= & \int_{0}^{T} \zeta \gamma\left\|\nabla\left(y_{i}^{n}-y_{i}\right)\right\|\left\|\Delta\left(y_{i}^{n}-y_{i}\right)\right\| d t \\
& +\left(\sup _{[0, T]} \gamma\left\|\nabla\left(y^{n}-y\right)\right\|\right)^{2} .
\end{aligned}
$$

Since $\epsilon_{i, n} \rightarrow 0$ as $n \rightarrow+\infty$ for $1 \leq i \leq 3$, this shows that 39 is satisfied.

Lemma 3.3. Let $\mathcal{A}$ be the mapping defined by 29]. Then $\mathcal{A}^{\prime}(0,0,0)$ is onto. 
Proof - Let us fix $\left(f, y_{0}\right) \in Z_{N}$. From Proposition 2.1 we know that there exists $(y, p, v)$ satisfying (18), (19) and (5). From the usual regularity results for the Stokes system, we have $y \in L^{2}(0, T ; D(A))$ and $p \in L^{2}\left(0, T ; H^{1}(\Omega)\right)$. Consequently, $(y, p, v) \in E_{N}$ and

$$
\mathcal{A}^{\prime}(0,0,0)(y, p, v)=\left(y_{t}-\nu \Delta y+\nabla p-v 1_{\omega}, y(\cdot, 0)\right)=\left(f, y_{0}\right) .
$$

This ends the proof.

In accordance with Lemmas 3.2 and 3.3 , we can apply Liusternik's Theorem (Theorem 3.1) and deduce that, at least in a neighborhood of the origin in $Z_{N}$, the equation

$$
\mathcal{A}(y, p, v)=\left(f, y_{0}\right), \quad(y, p, v) \in E_{N}
$$

possesses at least one solution.

Therefore, (1) is locally null-controllable and Theorem 1.1 is proved.

\section{The proof of Theorem 1.2}

We will now prove Theorem 1.2 . We will use an argument appropriate for quasi-Newton methods that rely on the $C^{1}$ regularity of $\mathcal{A}$; see for instance 2 .

From Section 2 we know that, for each $\left(f, y_{0}\right) \in Z_{N}$, the corresponding extremal problem (20) possesses exactly one solution. Let us consider the surjective operator $\mathcal{A}^{\prime}(0,0,0)$ and let us introduce the associated inverse $\mathcal{B}=$ $\mathcal{A}^{\prime}(0,0,0)^{-1}: Z_{N} \mapsto E_{N}$, defined as follows: for each $\left(f, y_{0}\right)$, we set $(y, p, v)=$ $\mathcal{B}\left(f, y_{0}\right)$ if and only if $(y, p, v)$ solves $20 p$.

Then, $\mathcal{B}$ is well defined and continuous. Indeed, as noticed in Section 2.2 , if $\left(f_{n}, y_{0 n}\right) \rightarrow\left(f, y_{0}\right)$ in $\left.Z_{N}, 21\right)$ holds. Using again the regularity properties of the solutions to the Stokes system, we also have

$$
y_{n} \rightarrow y \text { in } L^{2}(0, T ; D(A)) \text { and } p_{n} \rightarrow p \text { in } L^{2}\left(0, T ; H^{1}(\Omega)\right) .
$$

Consequently, $\left(y_{n}, p_{n}, v_{n}\right) \rightarrow(y, p, v)$, which means that $\mathcal{B}$ is continuous.

Let us consider the iterative algorithm ALG 1 introduced in Section 1 see (8). Then, for any initial $\left(y^{0}, p^{0}, v^{0}\right) \in E_{N}$, the iterates in ALG $\mathbf{1}$ are well defined.

Let us assume that $\left\|y_{0}\right\|_{H_{0}^{1}} \leq \epsilon(\epsilon$ is furnished by Theorem 1.1$)$ and $(y, p, v) \in$ $E_{N}$ satisfies $\mathcal{A}(y, p, v)=\left(0, y_{0}\right)$; let us set

$$
C_{0}:=\|\mathcal{B}\|_{\mathcal{L}\left(Z_{N} ; E_{N}\right)}
$$

and let us assume that $0<\bar{\theta}<1 /\left(2 C_{0}\right)$. Since $\mathcal{A}$ is continuously differentiable, there exists $\delta>0$ such that

$$
\|(\tilde{y}, \tilde{p}, \tilde{v})\|_{E_{N}},\left\|\left(y^{\prime}, p^{\prime}, v^{\prime}\right)\right\|_{E_{N}} \leq \delta \Rightarrow\left\|\mathcal{A}^{\prime}(\tilde{y}, \tilde{p}, \tilde{v})-\mathcal{A}^{\prime}\left(y^{\prime}, p^{\prime}, v^{\prime}\right)\right\|_{\mathcal{L}\left(E_{N} ; Z_{N}\right)} \leq \bar{\theta} .
$$

We will assume that $\|(y, p, v)\|_{E_{N}} \leq \delta$ and we will prove that there exists $\kappa>0$ such that, if $\left(y^{0}, p^{0}, v^{0}\right) \in E_{N}$ and

$$
\left\|\left(y^{0}, p^{0}, v^{0}\right)-(y, p, v)\right\|_{E_{N}} \leq \kappa,
$$


then $\left(y^{n}, p^{n}, v^{n}\right)$ converges to $(y, p, v)$ as $n \rightarrow+\infty$.

Let $\kappa$ be such that

$$
\begin{aligned}
(\tilde{y}, \tilde{p}, \tilde{v}) & \in E_{N}, \quad\|(\tilde{y}, \tilde{p}, \tilde{v})-(y, p, v)\|_{E_{N}} \leq \kappa \\
\Rightarrow & \left\{\begin{array}{c}
\left\|\mathcal{A}(\tilde{y}, \tilde{p}, \tilde{v})-\mathcal{A}(y, p, v)-\mathcal{A}^{\prime}(y, p, v)((\tilde{y}, \tilde{p}, \tilde{v})-(y, p, v))\right\|_{Z_{N}} \\
\leq \bar{\theta}\|(\tilde{y}, \tilde{p}, \tilde{v})-(y, p, v)\|_{E_{N}}
\end{array}\right.
\end{aligned}
$$

and let us choose $\left(y^{0}, p^{0}, v^{0}\right) \in E_{N}$ satisfying (41). Then, if we introduce $e^{n}:=$ $\left(y^{n}, p^{n}, v^{n}\right)-(y, p, v)$, the following holds:

$$
\begin{aligned}
e^{n+1}= & e^{n}-\mathcal{B}\left(\mathcal{A}\left(y^{n}, p^{n}, v^{n}\right)-\mathcal{A}(y, p, v)\right) \\
= & -\mathcal{B}\left(\mathcal{A}\left(y^{n}, p^{n}, v^{n}\right)-\mathcal{A}(y, p, v)-\mathcal{A}^{\prime}(0,0,0) e^{n}\right) \\
= & -\mathcal{B}\left(\mathcal{A}\left(y^{n}, p^{n}, v^{n}\right)-\mathcal{A}(y, p, v)-\mathcal{A}^{\prime}(y, p, v) e^{n}\right) \\
& -\mathcal{B}\left(\mathcal{A}^{\prime}(y, p, v)-\mathcal{A}^{\prime}(0,0,0)\right) e^{n} .
\end{aligned}
$$

Therefore,

$$
\begin{aligned}
\left\|e^{n+1}\right\|_{E_{N}} \leq & C_{0}\left\|\mathcal{A}\left(y^{n}, p^{n}, v^{n}\right)-\mathcal{A}(y, p, v)-\mathcal{A}^{\prime}(y, p, v) e^{n}\right\|_{Z_{N}} \\
& +C_{0}\left\|\mathcal{A}^{\prime}(y, p, v)-\mathcal{A}^{\prime}(0,0,0)\right\|_{\mathcal{L}\left(E_{N} ; Z_{N}\right)}\left\|e^{n}\right\|_{E_{N}} .
\end{aligned}
$$

Since $\left\|e^{0}\right\|_{E_{N}} \leq \kappa$, this inequality for $n=0$ yields

$$
\left\|e^{1}\right\|_{E_{N}} \leq 2 C_{0} \bar{\theta}\left\|e^{0}\right\|_{E_{N}}
$$

and, in particular, we also have $\left\|e^{1}\right\|_{E_{N}} \leq \kappa$. By induction, we then see that

$$
\left\|e^{n}\right\|_{E_{N}} \leq 2 C_{0} \bar{\theta}\left\|e^{n-1}\right\|_{E_{N}} \leq \cdots \leq\left(2 C_{0} \bar{\theta}\right)^{n}\left\|e^{0}\right\|_{E_{N}}
$$

for all $n \geq 1$. This proves that $e^{n} \rightarrow 0$ in $E_{N}$ and the inequalities (9) hold with $\theta=2 C_{0} \bar{\theta}$

This ends the proof.

\section{Numerical approximation and numerical ex- periments}

In this Section, we solve numerically the null controllability problem (1)(3) in a particular case and we check that the previous quasi-Newton iterates converge satisfactorily.

\subsection{Reformulation and numerical approximation of 20}

As indicated in Section 4 at each step of ALG 1 we have to find the solution to an extremal problem of the kind (20). In this Section, it will be shown that, in practice, this can be achieved by solving a high-order linear problem. 


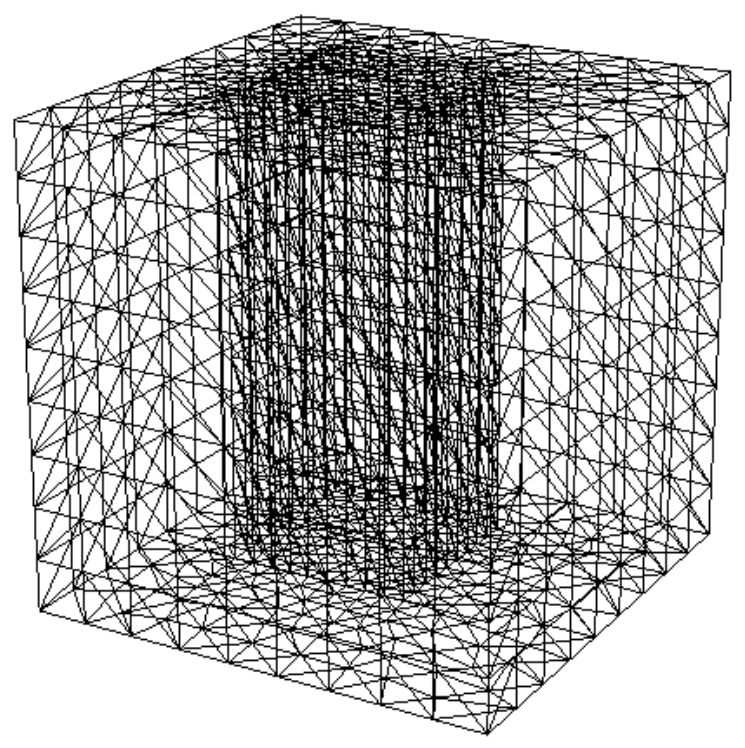

Figure 1: The domain $Q=\Omega \times(0, T)$ and the mesh. Number of vertices: 2800 . Number of tetrahedra: 14094 . Total number of unknowns: $8 \times 2800=22400$.

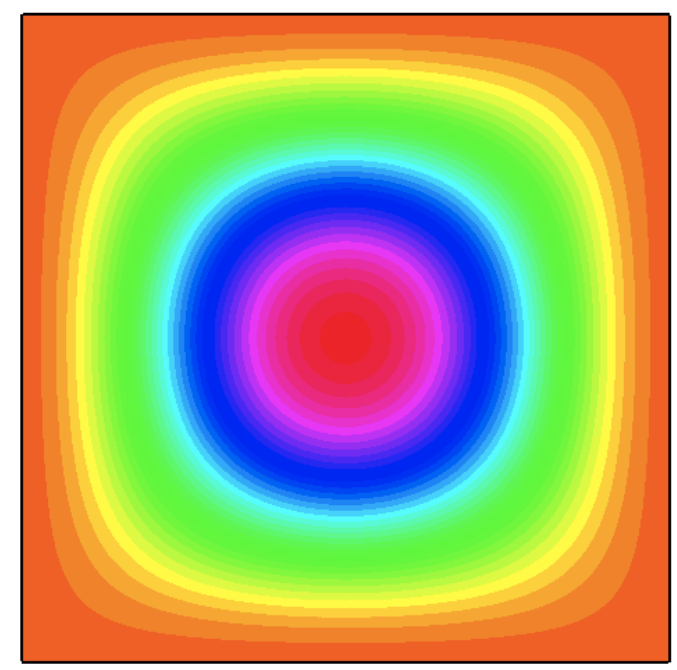

Figure 2: The initial state: the streamlines $\psi_{0}=$ Const., with $y_{0}=\nabla \times \psi_{0}$. 
More precisely, let us introduce the linear space

$$
P_{0}=\left\{(\zeta, \sigma) \in C^{2}(\bar{Q})^{N} \times C^{2}(\bar{Q}): \nabla \cdot \zeta=0 \text { in } Q, \zeta=0 \text { on } \Sigma, \int_{\Omega} \sigma d x=0 \text { a.e. }\right\}
$$

and let us denote by $P$ the completion of $P_{0}$ for the scalar product

$$
\begin{aligned}
\left((\zeta, \sigma),\left(\zeta^{\prime}, \sigma^{\prime}\right)\right)_{P} & :=\iint_{Q} \tilde{\rho}^{-2}\left(L^{*} \zeta+\nabla \sigma\right)\left(L^{*} \zeta^{\prime}+\nabla \sigma^{\prime}\right) d x d t \\
& +\iint_{\omega \times(0, T)} \tilde{\eta}^{-2} \zeta \zeta^{\prime} d x d t
\end{aligned}
$$

where we have used the notation $L^{*} \zeta:=-\zeta_{t}-\nu \Delta \zeta$. In view of 16) and the Carleman inequality $(15),(\cdot, \cdot)_{P}$ is a scalar product in $P_{0}$.

Another consequence of 15$)$ is that the functions $(\zeta, \sigma) \in P$ satisfy

$$
\begin{aligned}
& \iint_{Q} \rho_{*}^{-2}\left(s \xi_{*}\right)^{-1}\left(\left|\zeta_{t}\right|^{2}+|\nabla \sigma|^{2}\right) d x d t \\
& \quad+\iint_{Q} \tilde{\rho}^{-2}(s \tilde{\xi}) \lambda^{2}|\nabla \times \zeta|^{2} d x d t \\
& \quad+\iint_{Q} \tilde{\rho}^{-2}\left((s \tilde{\xi})^{-1}|\Delta \zeta|^{2}+\lambda^{2}|\nabla \zeta|^{2}+(s \tilde{\xi})^{2} \lambda^{4}|\zeta|^{2}\right) d x d t \\
& \quad<+\infty .
\end{aligned}
$$

In particular, one has

$$
\iint_{Q} \tilde{\eta}^{-2}|\zeta|^{2} d x d t<+\infty
$$

Also, $\zeta \in L^{2}(0, T / 2 ; D(A))$ and $\zeta_{t} \in L^{2}(0, T / 2 ; H)$, whence $\zeta \in C^{0}([0, T / 2] ; V)$, with appropriate estimates.

Let us assume that $\left(f, y_{0}\right)$ is given in $Z_{N}$ (recall that $\left.Z_{N}=L^{2}\left(\tilde{\eta}^{2} ; Q\right)^{N} \times V\right)$ and let us denote by $\Psi$ the following linear form on $P$ :

$$
\langle\Psi,(\zeta, \sigma)\rangle:=\iint_{Q} f \zeta d x d t+\int_{\Omega} y_{0}(x) \zeta(x, 0) d x .
$$

Then, $\Psi$ is well defined and continuous.

Therefore, there exists exactly one solution $(\zeta, \sigma)$ to the variational problem:

$$
\left\{\begin{array}{l}
\left((\zeta, \sigma),\left(\zeta^{\prime}, \sigma^{\prime}\right)\right)_{P}=\left\langle\Psi,\left(\zeta^{\prime}, \sigma^{\prime}\right)\right\rangle \\
\forall\left(\zeta^{\prime}, \sigma^{\prime}\right) \in P ; \quad(\zeta, \sigma) \in P .
\end{array}\right.
$$

The connection of 42 and 20 is explained in the following result:

Theorem 5.1. Let us assume that $\left(f, y_{0}\right) \in Z_{N}$ and let $(y, v)$ and $(\zeta, \sigma)$ be the unique solutions to the corresponding problems (20) and 42 . Then

$$
y:=\tilde{\rho}^{-2}\left(L^{*} \zeta+\nabla \sigma\right), \quad v:=-\left.\tilde{\eta}^{-2} \zeta\right|_{\omega \times(0, T)} .
$$


Proof - The argument relies on the ideas introduced by Fursikov and Imanuvilov in 20, that are adapted here to the case of the Stokes system (5).

Let $y$ and $v$ be given by 43, where $(\zeta, \sigma)$ is the unique solution to 42. Then

$$
J(y, v):=\frac{1}{2} \iint_{Q} \tilde{\rho}^{2}|y|^{2} d x d t+\frac{1}{2} \iint_{\omega \times(0, T)} \tilde{\eta}^{2}|v|^{2} d x d t<+\infty .
$$

and one has

$$
\begin{aligned}
& \iint_{Q} y \cdot\left(L^{*} \zeta^{\prime}+\nabla \sigma^{\prime}\right) d x d t=\iint_{\omega \times(0, T)} v \cdot \zeta^{\prime} d x d t \\
& \quad+\iint_{Q} f \cdot \zeta^{\prime} d x d t+\int_{\Omega} y_{0}(x) \cdot \zeta^{\prime}(x, 0) d x \quad \forall\left(\zeta^{\prime}, \sigma^{\prime}\right) \in P .
\end{aligned}
$$

Taking $\zeta^{\prime}=0$ and $\sigma^{\prime}$ arbitrary in $L^{2}\left(0, T ; H^{1}(\Omega)\right)$ with $\int_{\Omega} \sigma d x=0$ a.e., we first deduce from (44) that $y \in L^{2}(0, T ; H)$. Consequently,

$$
\begin{aligned}
& \iint_{Q} y \cdot L^{*} \zeta^{\prime} d x d t=\iint_{\omega \times(0, T)} v \cdot \zeta^{\prime} d x d t \\
& \quad+\iint_{Q} f \cdot \zeta^{\prime} d x d t+\int_{\Omega} y_{0}(x) \cdot \zeta^{\prime}(x, 0) d x \quad \forall\left(\zeta^{\prime}, \sigma^{\prime}\right) \in P .
\end{aligned}
$$

In particular, this must hold for any $\left(\zeta^{\prime}, 0\right)$ with $\zeta^{\prime} \in L^{2}(0, T ; D(A))$. This means that $y$ solves, in the transposition sense, the Stokes system (5).

But this system possesses exactly one strong solution. Therefore, $y$ is, together with some $p \in L^{2}\left(0, T ; H^{1}(\Omega)\right)$, the unique strong solution to (5).

Let $\left(y^{\prime}, v^{\prime}\right)$ be another state-control pair satisfying (5) (together with some $p^{\prime}$ ) and (19). In order to prove that $(y, v)$ solves 20), let us compare $J(y, v)$ and $J\left(y^{\prime}, v^{\prime}\right)$. We see that

$$
\begin{aligned}
J\left(y^{\prime}, v^{\prime}\right) & \geq J(y, v)+\iint_{Q} \tilde{\rho}^{2} y \cdot\left(y^{\prime}-y\right) d x d t+\iint_{\omega \times(0, T)} \tilde{\eta}^{2} v \cdot\left(v^{\prime}-v\right) d x d t \\
& =J(y, v)+\iint_{Q}\left(L^{*} \zeta+\nabla \sigma\right) \cdot\left(y^{\prime}-y\right) d x d t+\iint_{\omega \times(0, T)} \zeta \cdot\left(v^{\prime}-v\right) d x d t \\
& =J(y, v)+\iint_{Q} L^{*} \zeta \cdot\left(y^{\prime}-y\right) d x d t+\iint_{\omega \times(0, T)} \zeta \cdot\left(v^{\prime}-v\right) d x d t .
\end{aligned}
$$

The last identity holds because $y^{\prime}-y$ belongs to $L^{2}(0, T ; V)$.

However, since $y^{\prime}-y$ is (among other things) the solution in the transposition sense to the system (5) with $v$ and $\left(f, y_{0}\right)$ respectively replaced by $v^{\prime}-v$ and $(0,0)$, it is clear that

$$
\iint_{Q} L^{*} \zeta \cdot\left(y^{\prime}-y\right) d x d t+\iint_{\omega \times(0, T)} \zeta \cdot\left(v^{\prime}-v\right) d x d t=0 .
$$

Accordingly, $J\left(y^{\prime}, v^{\prime}\right) \geq J(y, v)$.

This ends the proof. 
Remark 5.1. Obviously, 42 can be equivalently rewritten as follows:

$$
\left\{\begin{array}{l}
\text { Minimize } \frac{1}{2}\|(\zeta, \sigma)\|_{P}^{2}-\langle\Psi,(\zeta, \sigma)\rangle, \\
\text { Subject to }(\zeta, \sigma) \in P
\end{array}\right.
$$

where $\|\cdot\|_{P}$ is the norm induced by $(\cdot, \cdot)_{P}$. It is not difficult to check that the extremal problems (20) and (45) are dual to each other in the sense of Fenchel-Rockafellar, see for instance $[12$. Both possess unique solutions and the optimality relations connecting them is just (43). Also, notice that $\zeta$ and $\sigma$ solve (together with some $p$ ), at least in a weak sense, the following partial differential system, that is fourth-order in space and second-order in time:

$$
\begin{cases}L\left(\tilde{\rho}^{-2}\left(L^{*} \zeta+\nabla \sigma\right)\right)+\nabla p+1_{\omega} \tilde{\eta}^{-2} \zeta=f(x, t), & (x, t) \in Q, \\ \nabla \cdot\left(\tilde{\rho}^{-2}\left(L^{*} \zeta+\nabla \sigma\right)\right)=0, \quad \nabla \cdot \zeta=0, & (x, t) \in Q, \\ \zeta=0, & (x, t) \in \Sigma, \\ \tilde{\rho}^{-2}\left(L^{*} \zeta+\nabla \sigma\right)=0, & (x, t) \in \Sigma, \\ \left.\tilde{\rho}^{-2}\left(L^{*} \zeta+\nabla \sigma\right)\right|_{t=0}=y_{0}(x),\left.\quad \tilde{\rho}^{-2}\left(L^{*} \zeta+\nabla \sigma\right)\right|_{t=T}=0, & x \in \Omega .\end{cases}
$$

There are several ways to introduce finite element approximations to 42. In the sequel, we will recall the main ideas of one of them; more details and other approaches can be found in [17]; see also 15, 16 for similar results in the context of the controlability of linear and semilinear heat equations.

For simplicity, it will be assumed in the following that $N=2$ and $\Omega$ is polygonal. Our approach needs a reformulation of 42 that is obtained in three steps:

1. First, let us introduce the new variables $z:=\tilde{\rho}^{-1}\left(L^{*} \zeta+\nabla \sigma\right), m:=\tilde{\eta}^{-1} \zeta$ and $\chi:=\tilde{\eta}^{-1} \sigma$ and the spaces

$$
S_{0}:=\left\{\left(\tilde{\eta}^{-1} \zeta, \tilde{\eta}^{-1} \sigma\right):(\zeta, \sigma) \in P\right\}, \quad \tilde{Z}:=L^{2}(Q)^{2} \times S_{0}, \quad \tilde{\Lambda}:=L^{2}(Q)^{2} .
$$

Then $(\zeta, \sigma) \in P$ if and only if $(z, m, \chi) \in \tilde{Z}$ and

$$
z-\tilde{\rho}^{-1}\left(L^{*}(\tilde{\eta} m)+\nabla(\tilde{\eta} \chi)\right)=0 .
$$

The latter is equivalent to

$$
\tilde{b}((z, m, \chi), \lambda):=\iint_{Q} \lambda\left(z-\tilde{\rho}^{-1}\left(L^{*}(\tilde{\eta} m)+\nabla(\tilde{\eta} \chi)\right) d x d t=0 \quad \forall \lambda \in \tilde{\Lambda} .\right.
$$

Therefore, 42 can be equivalently rewritten in the form

$$
\left\{\begin{array}{l}
a\left((z, m, \chi),\left(z^{\prime}, m^{\prime}, \chi^{\prime}\right)\right)+\tilde{b}\left(\left(z^{\prime}, m^{\prime}, \chi^{\prime}\right), \lambda\right)=\left\langle\tilde{\Psi},\left(z^{\prime}, m^{\prime}, \chi^{\prime}\right)\right\rangle \\
\tilde{b}\left((z, m, \chi), \lambda^{\prime}\right)=0 \\
\forall\left(z^{\prime}, m^{\prime}, \chi^{\prime}\right) \in \tilde{Z}, \forall \lambda^{\prime} \in \tilde{\Lambda} \\
(z, m, \chi) \in \tilde{Z}, \lambda \in \tilde{\Lambda}
\end{array}\right.
$$


where we have set

$$
a\left((z, m, \chi),\left(z^{\prime}, m^{\prime}, \chi^{\prime}\right)\right):=\iint_{Q}\left(z z^{\prime}+1_{\omega} m m^{\prime}\right) d x d t
$$

and

$$
\langle\tilde{\Psi},(z, m, \chi)\rangle:=\iint_{Q} \tilde{\eta} f m d x d t+\int_{\Omega} \tilde{\eta}(x, 0) y_{0}(x) m(x, 0) d x .
$$

Of course, $\lambda$ is a multiplier associated to the constraint (46). Note that the functions $\left(z^{\prime}, m^{\prime}, \chi^{\prime}\right) \in \tilde{Z}$ must satisfy $\nabla \cdot\left(\tilde{\eta} m^{\prime}\right) \equiv 0$.

2. Secondly, by integrating by parts, we can slightly change the bilinear form $\tilde{b}(\cdot, \cdot)$ and make disappear all the second-order derivatives in (47).

Thus, let us set

$$
\begin{gathered}
S_{1}:=\left\{(m, \chi): m \in L^{2}(0, T ; V), \chi \in L^{2}\left(0, T ; H^{1}(\Omega)\right),\right. \\
\left.m_{t} \in L^{2}(Q)^{2}, \int_{\Omega} \chi d x=0 \text { a.e. }\right\} \\
\hat{Z}:=L^{2}(Q)^{2} \times S_{1}, \quad \hat{\Lambda}:=L^{2}\left(0, T ; H_{0}^{1}(\Omega)^{2}\right)
\end{gathered}
$$

and

$\hat{b}((z, m, \chi), \lambda):=\iint_{Q}\left[\lambda\left(z+\tilde{\rho}^{-1}(\tilde{\eta} m)_{t}+\nabla(\tilde{\eta} \chi)\right)-\nabla\left(\tilde{\rho}^{-1} \lambda\right) \cdot \nabla(\tilde{\eta} m)\right] d x d t$.

Then, (47) can also be written as follows:

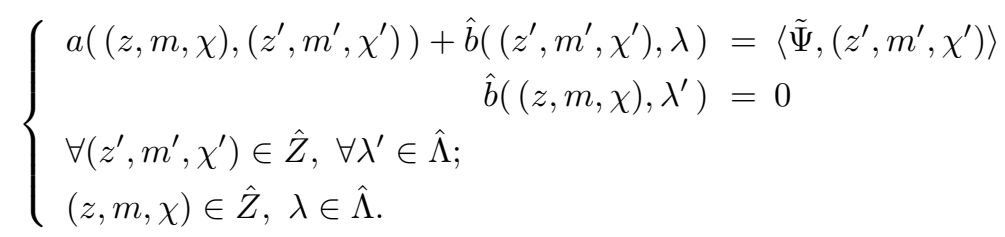

Notice that the new spaces $\hat{Z}$ and $\hat{\Lambda}$ satisfy

$$
\tilde{Z} \subset \hat{Z}, \quad \tilde{\Lambda} \supset \hat{\Lambda} .
$$

Again, the functions $\left(z^{\prime}, m^{\prime}, \chi^{\prime}\right) \in Z$ must satisfy $\nabla \cdot\left(\tilde{\eta} m^{\prime}\right) \equiv 0$.

3. In a third step, we introduce another multiplier, this time related to the constraint $\nabla \cdot(\tilde{\eta} m)=0$. Thus, we set

$$
\begin{gathered}
S_{2}:=\left\{(m, \chi): m \in L^{2}\left(0, T ; H_{0}^{1}(\Omega)^{2}\right), \chi \in L^{2}\left(0, T ; H^{1}(\Omega)\right),\right. \\
\left.m_{t} \in L^{2}(Q)^{2}, \int_{\Omega} \chi d x=0 \text { a.e. }\right\}
\end{gathered}
$$




$$
W:=L^{2}(Q)^{2} \times S_{2}, \quad M:=\hat{\Lambda} \times L^{2}(Q)
$$

and

$$
B((z, m, \chi),(\lambda, \mu)):=\hat{b}((z, m, \chi), \lambda)-\iint_{Q} \tilde{\rho}^{-1} \mu \nabla \cdot(\tilde{\eta} m) d x d t
$$

and we arrive at the following reformulation:

$$
\left\{\begin{array}{l}
a\left((z, m, \chi),\left(z^{\prime}, m^{\prime}, \chi^{\prime}\right)\right)+B\left(\left(z^{\prime}, m^{\prime}, \chi^{\prime}\right),(\lambda, \mu)\right)=\left\langle\tilde{\Psi},\left(z^{\prime}, m^{\prime}, \chi^{\prime}\right)\right\rangle \\
B\left((z, m, \chi),\left(\lambda^{\prime}, \mu^{\prime}\right)\right)=0 \\
\forall\left(z^{\prime}, m^{\prime}, \chi^{\prime}\right) \in W, \quad \forall\left(\lambda^{\prime}, \mu^{\prime}\right) \in M ; \\
(z, m, \chi) \in W, \quad(\lambda, \mu) \in M .
\end{array}\right.
$$

This way, we are led to a mixed formulation of 42 where the exponential weights have disappeared. It will be seen later that, at the end, this property has a positive numerical effect: after time-space approximation, we find linear systems for which the condition numbers are moderate; for more details on this phenomenon, see [15, 17.

A good property of 49 is that the involved bilinear and linear forms are well defined in spaces of functions that possess first-order (and not necessarily second-order) derivatives in $L_{\mathrm{loc}}^{2}(Q)$. Another advantage is that, now, the unknown $m$ is not subject to any incompressibility constraint.

Accordingly, it is easy to construct finite dimensional subspaces of $W$ and $M$, for instance using the continuous piecewise polynomial functions associated to a mesh of $Q=\Omega \times(0, T)$.

\begin{tabular}{|c|c|c|}
\hline Iterate & Abs. error & Rel. error \\
\hline 1 & 13.4761 & 13.4761 \\
\hline 2 & 0.61022 & 0.0452815 \\
\hline 3 & 0.022168 & 0.00164313 \\
\hline 4 & 0.00101488 & $7.52252 \times 10^{-5}$ \\
\hline 5 & $4.9267 \times 10^{-5}$ & $3.65175 \times 10^{-6}$ \\
\hline \hline
\end{tabular}

Table 1: The behavior of ALG 1.

More precisely, let $\mathcal{T}_{h}$ be a triangulation of $Q$ formed by tetrahedra and let $W_{h}$ (resp. $M_{h}$ ) be the subspace of $W$ (resp. $M$ ) formed by the usual $P_{\ell}$-Lagrange functions $(\ell=1$ or $\ell=2)$. Obviously, the $W_{h}$ and $M_{h}$ are finite dimensional spaces and it is well known that they are easy to describe and manipulate.

The approximated problem is the following:

$$
\left\{\begin{array}{l}
a\left((z, m, \chi),\left(z^{\prime}, m^{\prime}, \chi^{\prime}\right)\right)+B\left(\left(z^{\prime}, m^{\prime}, \chi^{\prime}\right),(\lambda, \mu)\right)=\left\langle\tilde{\Psi},\left(z^{\prime}, m^{\prime}, \chi^{\prime}\right)\right\rangle \\
B\left((z, m, \chi),\left(\lambda^{\prime}, \mu^{\prime}\right)\right)=0 \\
\forall\left(z^{\prime}, m^{\prime}, \chi^{\prime}\right) \in W_{h}, \quad \forall(\lambda, \mu) \in M_{h} ; \\
(z, m, \chi) \in W_{h}, \quad(\lambda, \mu) \in M_{h} .
\end{array}\right.
$$




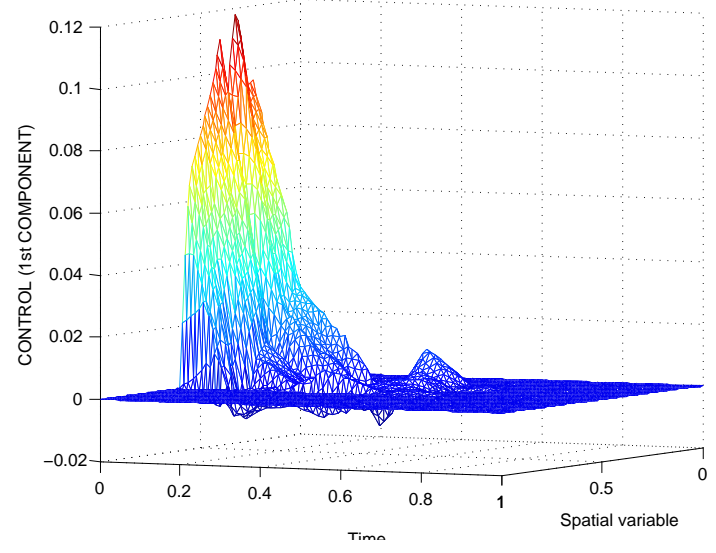

Figure 3: Cut section of $v_{1}$ (the first component of the control) at $x_{1}=0.4$; $t$ goes from left to right, $x_{2}$ goes from top to botttom; minimal and maximal values of $v_{1}:-0.0173$ and 0.1205 . 


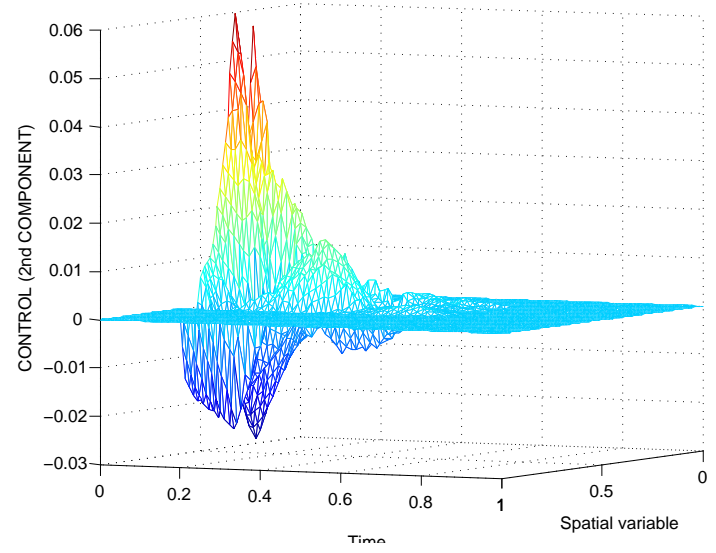

Figure 4: Cut section of $v_{2}$ (the second component of the control) at $x_{1}=0.4$; $t$ goes from left to right, $x_{2}$ goes from top to botttom; minimal and maximal values of $v_{2}:-0.0285$ and 0.0607 . 


\subsection{A numerical experiment}

The following computations have been performed with the FreeFem ++ library; see http://www.freefem.org/ff++ for a detailed description. In particular, the solutions to the linear systems $(50)$ have been computed by a $U M F$ $P A C K$ solver, specially adequate for sparse unsymmetric large dimension matrices of this kind.

The quasi-Newton method has been applied to the solution of the null controllability problem for (1) with the following data:

- $N=2, \Omega=(0,1) \times(0,1), \omega=(0.2,0.6) \times(0.2,0.6), T=1$.

- $y_{0}=\nabla \times \psi_{0}$, with $\psi_{0}(x) \equiv 10^{4} x_{1}^{2}\left(1-x_{1}\right)^{2} x_{2}^{2}\left(1-x_{2}\right)^{2}$.

- $\nu_{0}=10, \nu_{1}(r):=(1+r)^{-1}$ and consequently $\nu=10$.

At each step, we have solved the problem (50). Here, the spaces $W_{h}, \Lambda_{h}$ and $M_{h}$ are $P_{1}$-Lagrange approximations respectively of $W_{h}, \Lambda_{h}$ and $M_{h}$, associated to the mesh displayed in Fig. 5 .

The initial state is displayed in Fig. 5 .

The stopping criterion has been

$$
\left\|y^{n+1}-y^{n}\right\| \leq \epsilon
$$

with $\epsilon=10^{-5}$. Starting from $\left(y^{0}, z^{0}, v^{0}\right)=\mathcal{B}\left(0, y_{0}\right)$, convergence was reached after 5 iterates, with a convergence rate $\approx 4.45$. The absolute and relative errors at each step are given in Table 1. The numerical control and state that solve (1) - (3) are displayed in Fig. 5.1 6 .

The condition numbers of the matrices of coefficients in the linear systems that must be solved are given in Table 2 for several meshes. As already mentioned, the fact that we work with the variables $z, m, \chi$, etc. lead to reasonable quantities.

\begin{tabular}{|c|c|c|}
\hline Number of nodes & Number of unkowns & Cond. number \\
\hline 305 & 2440 & $9.91 \times 10^{5}$ \\
\hline 960 & 7680 & $2.02 \times 10^{6}$ \\
\hline 2800 & 22400 & $1.31 \times 10^{6}$ \\
\hline
\end{tabular}

Table 2: Condition numbers of the matrices of coefficients in 50 for several meshes.

\section{Some additional comments and questions}

The global null controllability of (1) is an open question. It does not seem easy to solve. Indeed, the smallness assumption on the data in Theorem 1.1 is clearly necessary if one tries to apply Theorem 3.1 or another result playing 


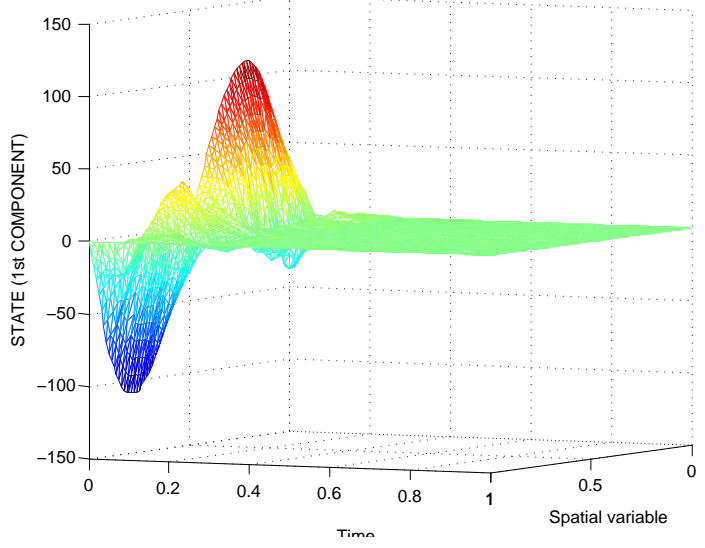

Figure 5: Cut section of $y_{1}$ (the first component of the state) at $x_{1}=0.4 ; t$ goes from left to right, $x_{2}$ goes from top to botttom; minimal and maximal values of $y_{1}:-111.1570$ and 112.7190 . 


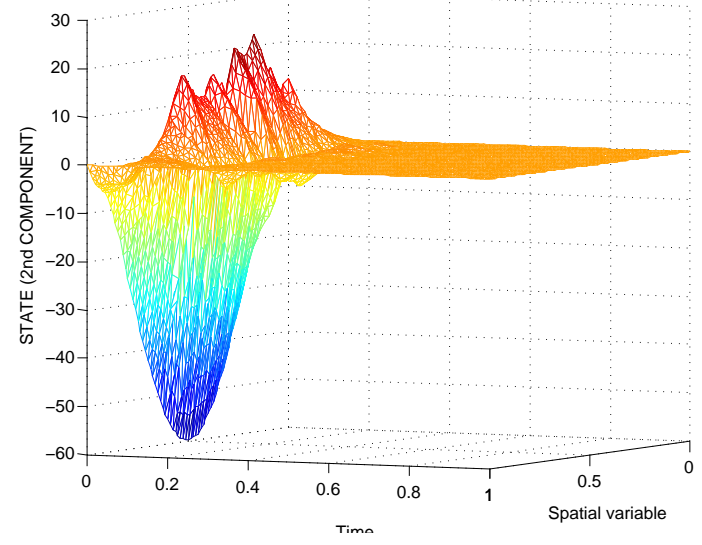

Figure 6: Cut section of $y_{2}$ (the second component of the state) at $x_{1}=0.4$; $t$ goes from left to right, $x_{2}$ goes from top to botttom; minimal and maximal values of $y_{2}$ : -60.6470 and 25.1871 . 
the same role. To prove a global result, we would have to make use of a global inverse mapping theorem, but this needs much more complicate estimates, that do not seem affordable.

Notice that, with other or with no boundary conditions, global null controllability results have been established for Navier-Stokes and Boussinesq fluids by Coron $[7$ and Coron and Fursikov $\underline{9}$ in the two-dimensional case and Fursikov and Imanuvilov 21 in the three-dimensional case. Accordingly, it is reasonable to expect results of the same kind when the PDEs in (1) are completed, for instance, with Navier-slip or periodic boundary conditions.

Another open question, in part connected to the previous one, concerns the exact controllability to the trajectories.

It is said that (1) is locally exactly controllable to the trajectories at time $T$ if, for any solution $(\hat{y}, \hat{p})$ corresponding to a control $\hat{v}$, there exists $\epsilon>0$ such that, if

$$
\left\|y_{0}-\hat{y}(\cdot, 0)\right\|_{H_{0}^{1}} \leq \epsilon
$$

we can find controls $v \in L^{2}(\omega \times(0, T))$ and associated states $(y, p)$ satisfying

$$
y(x, T)=\hat{y}(x, T) \text { in } \Omega .
$$

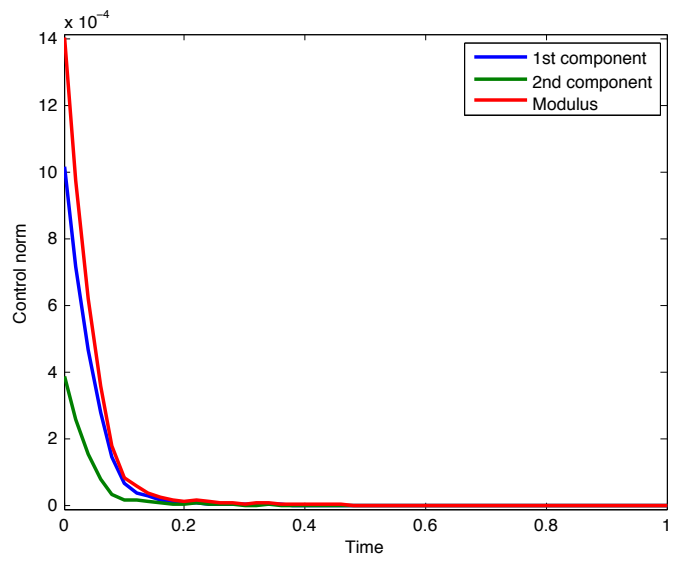

Figure 7: The computed null control; evolution in time of the $L^{2}$ norms of $v_{1}$, $v_{2}$ and $v$.

The previous property was established for Navier-Stokes and Boussinesq fluids in [14. However, to our knowledge, it is unknown whether it holds for (1). If one tries to apply arguments as those above, one finds at once a major difficulty: one is led to a system similar to (6) where linear nonlocal terms appear, for which observability estimates are not clear at all.

Finally, let us indicate that it would be interesting to see whether the arguments in 4 can be applied in this context to establish the local null controllability of $(1)$ with $N-1$ scalar controls; when $N=3$, a similar question arises 


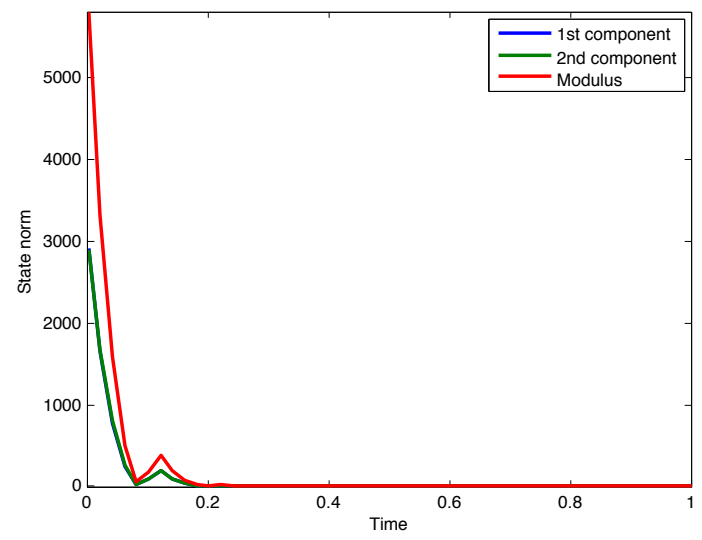

Figure 8: The computed state; evolution in time of the $L^{2}$ norms of $y_{1}, y_{2}$ and $y$.

concerning the controllability of (1) with one single scalar control, in view of the results in [10].

\section{Appendix A: Existence and uniqueness results for (1)}

Let us set $\nu(s):=\nu_{0}+\nu_{1}(s)$. The existence of a strong solution to (1) can be proved as follows.

First, we introduce the eigenfunctions of the Stokes operator, i.e. the solutions to

$$
\begin{cases}-\Delta w^{j}+\nabla q^{j}=\lambda_{j} w^{j} & \text { in } \Omega \\ w^{j}=0 & \text { on } \partial \Omega \\ \left\|w^{j}\right\|=1, \quad \lambda_{j} \rightarrow+\infty, & \end{cases}
$$

the spaces $V_{m}:=\left[w^{1}, \ldots, w^{m}\right]$ and the following associated Galerkin approximations

$$
\left\{\begin{array}{l}
\left(y_{m}^{\prime}, w\right)+\nu\left(\left\|D y_{m}\right\|^{2}\right)\left(\nabla y_{m}, \nabla w\right)+\left(\left(y_{m} \cdot \nabla\right) y_{m}, w\right)=\left(v 1_{\omega}, w\right) \quad \forall w \in V_{m}, \\
y_{m}:[0, T] \mapsto V_{m}, \quad y(0)=y_{0 m},
\end{array}\right.
$$

where $y_{0 m} \rightarrow y_{0}$ in $V$.

The existence and uniqueness of (local in time) solutions to the 52 is ensured by classical ODE theory. The following estimates show that, in fact, they are defined for all $t$.

We can get uniform estimates of the $y_{m}$ in the usual way: 
- First, taking $w=y_{m}(t)$, we deduce that

$$
\begin{gathered}
\frac{1}{2} \frac{d}{d t}\left\|y_{m}\right\|^{2}+\nu\left(\left\|D y_{m}\right\|^{2}\right)\left\|\nabla y_{m}\right\|^{2}=\left(v 1_{\omega}, y_{m}\right) \\
\leq C\left\|y_{m}\right\|^{2}+C \int_{\omega}|v|^{2} d x
\end{gathered}
$$

whence we easily obtain

$$
\left\|y_{m}\right\|_{L^{\infty}(0, T ; H)}+\left\|y_{m}\right\|_{L^{2}(0, T ; V)} \leq C
$$

- Then, noticing that $A y_{m}(t) \in V_{m}$ and taking $w=A y_{m}(t)$, we see that

$$
\frac{1}{2} \frac{d}{d t}\left\|\nabla y_{m}\right\|^{2}+\nu\left(\left\|D y_{m}\right\|^{2}\right)\left\|\Delta y_{m}\right\|^{2}=\left(v 1_{\omega}, A y_{m}\right)-\left(\left(y_{m} \cdot \nabla\right) y_{m}, A y_{m}\right) \text {. }
$$

When $N=2$, one has

$$
\begin{aligned}
\left(\left(y_{m} \cdot \nabla\right) y_{m}, A y_{m}\right) & \leq C\left\|y_{m}\right\|_{L^{4}}\left\|\nabla y_{m}\right\|_{L^{4}}\left\|A y_{m}\right\| \\
& \leq C\left\|y_{m}\right\|^{2}\left\|\nabla y_{m}\right\|^{2}+\frac{\nu_{0}}{2}\left\|A y_{m}\right\|^{2} .
\end{aligned}
$$

Consequently,

$$
\frac{1}{2} \frac{d}{d t}\left\|\nabla y_{m}\right\|^{2}+\left\|\Delta y_{m}\right\|^{2} \leq C\left\|\nabla y_{m}\right\|^{2}+C \int_{\omega}|v|^{2} d x
$$

and, from Gronwall's Lemma, we find that

$$
\left\|y_{m}\right\|_{L^{\infty}(0, T ; V)}+\left\|y_{m}\right\|_{L^{2}(0, T ; D(A))} \leq C .
$$

When $N=3$, the nonlinear term can be bounded as follows:

$$
\begin{aligned}
\left(\left(y_{m} \cdot \nabla\right) y_{m}, A y_{m}\right) & \leq C\left\|y_{m}\right\|_{L^{6}}\left\|\nabla y_{m}\right\|_{L^{3}}\left\|A y_{m}\right\| \\
& \leq C\left\|\nabla y_{m}\right\|^{3 / 2}\left\|A y_{m}\right\|^{3 / 2} \\
& \leq C\left\|\nabla y_{m}\right\|^{6}+\frac{\nu_{0}}{2}\left\|A y_{m}\right\|^{2} .
\end{aligned}
$$

Accordingly, we only have in this case that

$$
\frac{1}{2} \frac{d}{d t}\left\|\nabla y_{m}\right\|^{2}+\left\|\Delta y_{m}\right\|^{2} \leq C\left\|\nabla y_{m}\right\|^{6}+C \int_{\omega}|v|^{2} d x
$$

and, if $y_{0}$ and $v$ are sufficiently small respectively in $V$ and $L^{2}(\omega \times(0, T)$, we get again (54).

- From these estimates, we see that

$$
\nu\left(\left\|D y_{m}\right\|^{2}\right) A y_{m}-\left(y_{m} \cdot \nabla\right) y_{m}+v 1_{\omega}
$$

is uniformly bounded in $L^{2}(Q)^{N}$. In view of 52 and the fact that the $w^{j}$ are orthonormal in $L^{2}(\Omega)^{N}$, we deduce that

$$
\left\|y_{m}^{\prime}\right\|_{L^{2}(Q)^{N}} \leq C .
$$


The uniform bounds (53)- 55 allow to tke limits in 52 (at least for a subsequence) as $m \rightarrow+\infty$. Indeed, the unique delicate point is the a.e. convergence of $\nu\left(\left\|D y_{m}\right\|^{2}\right)$. But this is a consequence of the fact that the sequence $\left\{y_{m}\right\}$ is pre-compact in $L^{2}(0, T ; V)$ and $\nu$ is continuous.

The uniqueness of the strong solution to (1) can be proved in a standard way. Indeed, if $\left(y^{1}, p^{1}\right)$ and $\left(y^{2}, p^{2}\right)$ are strong solutions and we set $y:=y^{1}-y^{2}$, we find that

$$
\begin{aligned}
& \frac{1}{2} \frac{d}{d t}\|\nabla y\|^{2}+\nu\left(\left\|D y^{1}\right\|^{2}\right)\|\Delta y\|^{2} \\
& \quad=-\left(\nu\left(\left\|D y^{1}\right\|^{2}\right)-\nu\left(\left\|D y^{2}\right\|^{2}\right)\right)\left(\nabla y^{2},-\Delta y\right)-\left(\left(y^{1} \cdot \nabla\right) y+(y \cdot \nabla) y^{2},-\Delta y\right) \\
& \quad \leq C\left[\left(\left\|\nabla y^{1}\right\|+\left\|\nabla y^{2}\right\|\right)\left\|\nabla y^{2}\right\|+\left\|\Delta y^{1}\right\|+\left\|\Delta y^{2}\right\|\right]\|\Delta y\|\|\nabla y\| \\
& \quad \leq k(t)\|\nabla y\|^{2}+\frac{\nu_{0}}{2}\|\Delta y\|^{2}
\end{aligned}
$$

for some $k \in L^{2}(0, T)$. This suffices to deduce that $y \equiv 0$.

\section{Appendix B: Proof of the Carleman estimate 12}

First, note that there exists $\lambda_{0}$ such that, for any $\lambda \geq \lambda_{0}$, there exist $s_{0}$ and $C$ with

$$
\begin{aligned}
\iint_{Q} \rho^{-2}(s \xi)|\nabla \times \varphi|^{2} d x d t & +\iint_{Q} \rho^{-2}(s \xi)^{-1}|\nabla(\nabla \times \varphi)|^{2} d x d t \\
& +\iint_{Q} \rho^{-2}\left(|\nabla \varphi|^{2}+(s \xi)^{2}|\varphi|^{2}\right) d x d t \\
\leq & C\left(\iint_{Q} \rho^{-2}|F|^{2} d x d t+\iint_{\omega \times(0, T)} \rho^{-2}(s \xi)^{3}|\varphi|^{2} d x d t\right)
\end{aligned}
$$

for any $F \in L^{2}(Q)^{N}$, any $\varphi_{T} \in H$ and any $s \geq s_{0}$.

This is established in Theorem 4.1 in [26]; see also Theorem 3.1 in 25 .

Then, we argue as follows:

- Since $\nabla \cdot \varphi \equiv 0$, the second order term $\Delta \varphi$ satisfies

$$
|\Delta \varphi|^{2}=|\nabla \times(\nabla \times \varphi)|^{2} \leq 2|\nabla(\nabla \times \varphi)|^{2} .
$$

This, together with 56 , provides a similar bound for $|\Delta \varphi|^{2}$ :

$$
\iint_{Q} \rho^{-2}(s \xi)^{-1}|\Delta \varphi|^{2} d x d t \leq C\left(\iint_{Q} \rho^{-2}|F|^{2} d x d t+\iint_{\omega \times(0, T)} \rho^{-2}(s \xi)^{3} \lambda^{4}|\varphi|^{2} d x d t\right) .
$$


- On the other hand, one has

$$
-\varphi_{t}+\nabla \pi=F+\nu \Delta \varphi \text { in } Q .
$$

Using again that $\varphi$ is divergence-free and the fact that $\bar{\rho}$ and $\bar{\xi}$ are independent of $x$, we see that

$$
\begin{gathered}
\iint_{Q} \bar{\rho}^{-2}(s \bar{\xi})^{-1}\left(\left|\varphi_{t}\right|^{2}+|\nabla \pi|^{2}\right) d x d t=\iint_{Q} \bar{\rho}^{-2}(s \bar{\xi})^{-1}\left|-\varphi_{t}+\nabla \pi\right|^{2} d x d t \\
\quad \leq C \iint_{Q} \rho^{-2}(s \xi)^{-1}\left(|F|^{2}+|\Delta \varphi|^{2}\right) d x d t
\end{gathered}
$$

whence the estimates of $\left|\varphi_{t}\right|^{2}$ and $|\nabla \pi|^{2}$ in $(12)$ are also obtained.

This ends the proof.

\section{Acknowledgements}

The authors are indebted to the anonymous referees for their comments and suggestions, that have lead to a considerable improvement of this paper.

\section{References}

[1] V.M. Alekseev, V.M. Tikhomorov, S.V. Formin, Optimal control, Contemporary Soviet Mathematics, Consultants Bureau, New York, 1987.

[2] I.K. Argyros, Convergence and applications of Newton-type iterations, Springer, New York, 2008.

[3] L.C. Berselli, T. Iliescu, W.J. Layton, Mathematics of large eddy simulation of turbulent flows, Scientific Computation, Springer-Verlag, Berlin, 2006.

[4] N. Carreño, S. Guerrero, Local null controllability of the $N$-dimensional Navier-Stokes system with $N-1$ scalar controls in an arbitrary control domain, J. Math. Fluid Mech. 15 (2013), no. 1, 139-153.

[5] T. Chacón, R. Lewandowski, Mathematical and numerical foundations of turbulence models and applications, Springer, to appear.

[6] H. Clark, E. Fernández-Cara, J. Limaco, L. A. Medeiros, Theoretical and numerical local null controllability for a parabolic system with local and nonlocal nonlinearities, Applied Math. Computation 223 (2013), 483-505.

[7] J.-M. Coron, On the controllability of the 2-D incompressible Navier?Stokes equations with the Navier slip boundary conditions, ESAIM Control Optim. Calc. Var. 1 (1996) $\underline{35-75 .}$

[8] J.-M. Coron, Control and nonlinearity, Mathematical surveys and monographs, v. 136, American Mathematical Society, Rhode Island, USA, 2007.

[9] J.-M. Coron, A. V. Fursikov, Global exact controllability of the 2D Navier-Stokes equations on a manifold without boundary, Russian J. Math. Phys. v. 4, No. 4, 1996, p. 429$\underline{448 .}$ 
[10] J.-M. Coron, P. Lissy, Local null controllability of the three-dimensional Navier-Stokes system with a distributed control having two vanishing components, to appear in Inventiones Math.

[11] P. Constantin, C. Foias, Navier-Stokesequations, Chicago Lectures in Mathematics, University of Chicago Press, 1988.

[12] I. Ekeland, R. Témam, Convex analysis and variational problems, Classics in Applied Mathematics, 28, Society for Industrial and Applied Mathematics (SIAM), Philadelphia, PA, 1999.

[13] C. Fabre, J.-P. Puel, E. Zuazua, Approximate controllability of the semilinear heat equation, Proc. Royal Soc. Edinburgh, 125A (1995), 31-61.

[14] E. Fernández-Cara, S. Guerrero, O. Yu. Imanuvilov, J.-P. Puel, Local exact of the NavierStokes systems, J. Math. Pures Appl., 83/12 (2004), 1501-1542.

[15] E. Fernández-Cara, A. Münch, Strong convergent approximations of null controls for the 1D heat equation, SeMA J. (2013) 61:49-78.

[16] E. Fernández-Cara, A. Münch, Numerical null controllability of semi-linear 1D heat equations: fixed point, least squares and Newton methods, Math. Control and Related Fields, Vol. 2, No. 3, September 2012.

[17] E. Fernández-Cara, A. Münch, D.A. Souza, Numerical null controllability of Stokes and Navier-Stokes equations, in preparation.

[18] A. V. Fursikov, Exact boundary zero controllability of three-dimensional Navier-Stokes equations, J. of Dynamical and Control Systems., v. 1, No. 3 (1995), p. 325-350.

[19] A.V. Fursikov, O.Yu. Imanuvilov, On exact boundary zero controllability of twodimensional Navier-Stokes equations, Acta Applic. Math. v. 37, 1994, p. 67-76.

[20] A. V. Fursikov, O. Yu. Imanuvilov, Controllability of Evolution Equations, Lecture Notes Series, Vol. 34, Seoul National University, Research Institute of Mathematics, Global Analysis Research Center, Seoul, 1996.

[21] A. V. Fursikov, O. Yu. Imanuvilov, Exact controllability of the Navier-Stokes and Boussinesq equations, Russian Math. Surveys, vol. 54:3 (1999), 565-618.

[22] M. González-Burgos, S. Guerrero, J.-P. Puel, Local exact controllability to the trajectories of the Boussinesq system via a fictitious control on the divergence equation, Comm. Pure and Applied Analysis, Vol. 8, No. 1, January 2009.

[23] J.-L. Guermond, J. T. Oden, S. Prudhomme, Mathematical Perspectives on Large Eddy Simulation Models for Turbulent Flows, J. math. fluid mech. 6 (2004) 194-248.

[24] O. Yu. Imanuvilov, Controllability of parabolic equations (Russian), Mat. Sbornik. Novaya Seriya, 186 (1995), 109-132.

[25] O. Yu. Imanuvilov, J.-P. Puel, M. Yamamoto, Carleman estimates for parabolic equations with nonhomogeneous boundary conditions, Chin. Ann. Math, Ser. B 30 (2009), no. $4,333-378$.

[26] O. Yu. Imanuvilov, J.-P. Puel, M. Yamamoto, Carleman estimates for second order non homogeneous parabolic equations, preprint, 2010.

[27] B.E. Launder, W.C. Reynolds, W. Rodi, Turbulence models and their applications, Vol. 2, Collection de la Direction des Études et Recherches d'Électricité de France ESE, 56, Éditions Eyrolles, Paris, 1984. 
[28] M. Lesieur, Turbulence in Fluids, (Third Edition), Dordrecht: Kluwer Academic Publishers, 1997.

[29] T.L. Saaty, Modern nonlinear equations, Corrected reprint of the 1967 original, Dover Books on Advanced Mathematics, Dover Publications, Inc., New York, 1981.

[30] M. Tucsnak, G. Weiss, Observation and control for operator semigroups, Birkhäuser Advanced Texts: Basler Lehrbücher, Birkhäuser Verlag, Basel, 2009.

[31] E. Zuazua, Exact boundary controllability for the semilinear wave equation, Nonlinear Partial Differential Equations and their Applications, 10 (1989), 357-391.

[32] E. Zuazua, Controllability and observability of partial differential equations: some results and open problems, Handbook of differential equations: evolutionary equations, Vol. III, 527-621, Handb. Differ. Equ., Elsevier/North-Holland, Amsterdam, 2007. 